\title{
COMPARISON OF MICROSCOPIC AND MACROSCOPIC INSTABILITIES IN A CLASS OF TWO-DIMENSIONAL PERIODIC COMPOSITES
}

\author{
Nicolas Triantafyllidis and William C. Schnamt \\ Aerospace Engineering, University of Michigan, Am Arbor, MI 48109-2I40, U.S.A.
}

(Received 15 October 1992)

\begin{abstract}
OF INTEREST in the present work is the quantitative comparison between the microscopic and the corresponding macroscopic failure in a two-dimensional elastic medium with known periodic microstructure. This comparison establishes theoretical limits for the validity for the averaged (homogenized) response of microstructured elastic media. Attention is focussed on an approximation of a fiber reinforced composite modeled as an infinite periodic grillage of axially compressed beams with an average shear stiffness. The particular beam model was chosen so that the problem would be analytically tractable, yet of sufficient cemplexity to exhibit nontrival micoscopic and macruscopic failure nodes.

A comparison of the stresses at the onset of the microscopic falure (taken to be the first bifurcation away from the principal periodic solution) to the stresses at the onset of the macroscopic fallure (which corresponds to the loss of ellipticity for the incremental response of the homogenized model) allows one to identify whether the first failure mode, also termed the critical mode, is local (microscopic) or global (macroscopic) in nature. An extensive investigation of the influence of various model parameters on the failure modes of the composite has been undertaken. The results obtained show the importance of the interstitial stiffness in deciding the nature of the critical mode. The presentation is concluded by a discussion of the results with suggestions for future work.
\end{abstract}

\section{INTRODUCTION}

ONE OF THE MOST INTERESTING questions in mechanics pertains to the study of stability and failure of media with microstructure. This problem is an important aspect of the broader issue of change of scale for problems in mechanics, where one wants to predict the macroscopic behavior of a medium based on knowledge of its microstructure. Since some kind of instability induced failure is inevitable at adequately high levels of loading, of interest here is the quantitative comparison between the onset of the first microscopic instability in the medium and the onset of the corresponding instability predicted by the macroscopic equations for the medium. Such comparisons establish the theoretical limits of validity for the averaged models of the microstructured solids.

A technologically important problem that belongs to the above general class of problems in mechanics pertains to the stability of composite media. Customarily a composite medium is idealized by a homogeneous continuum whose properties have been obtained by using an appropriate averaging technique (e.g. the homogenization method, a self-consistent type method) which takes into account the solid's micro- 
structure. For inelastic materials, the averaged homogeneous continua include in their description internal variables which are directly related to certain features of the microstructure (such as porosity, crack shape and orientation, and so on). The question of the composite's stability is usually addressed by investigating the (homogeneous) macroscopic properties of the solid, and the onset of instability is often signaled by some internal variable reaching a critical value. On the other hand, instability in composites can be analysed directly from the knowledge of the microstructure in a direct approach which is also frequently followed in studies of fallure mechanisms for composites.

Due to the complex, often irregular geometries of microstructures and the rather involved nature of constitutive equations in real composites, one faces severe difficulties in identifying a clear cut onset of instability or failure in composites at both the micro and macro level. The construction of the macroscopic model requires a variety of often difficult to verify intuitive assumptions and so does the study of the stability of the solid at the microscopic level. Hence, few attempts have been made thus far to correlate the stability predictions of a consistently derived macroscopic model to those obtained by analysing the exact microstructural instability mechanism for a given composite.

In order to simplify the problem so as to permit a consistent derivation of the macroscopic behavior of the solid, attention is focussed on periodic microgeometries, created by the repetition of a fundamental building block called the unit cell. Also in the interest of simplicity, only rate independent materials are being considered. One of the first studies in this direction, for finitely strained solids, was presented by TVERGAARD (1981) who investigated the effects of voids on shear band formation in finitely strained periodic porous material. He compared the microscopic buckling loads and modes to the predictions for shear band instabilities based on an approximate macroscopic model for the void-matrix aggregate. Subsequently, ArFyaratnF: and TRIANTAFYllidis (1984) used a consistent homogenization theory approach (needed to model correctly the strong interactions between the voids) to study macroscopic instability, manifested as a loss of ellipticity (i.e. emergence of shear bands), for a finitely strained periodic porous elastic solid.

The loss of ellipticity of the equations at the macroscopic level is due to a bifurcation of the microstructure away from the periodic principal solution whose period is the same as the one for the unit cell. This explains the macroscopic behavior exhibited by the models in the above mentioned work by ABEYARATNE and TRIANTAFYllidis (1984). Hence, for the class of finitely strained rate independent solids with periodic microstructures there exists a clear definition for the onset of the microscopic instability (bifurcation away from the periodic single cell principal solution) and a corresponding definition for the onset of the macroscopic instability (emergence of shear bands). Moreover, one can show that the microscopic bifurcation is responsible for the macroscopic loss of ellipticity. The latter phenomenon can be captured from the former when the buckling mode at the microscopic level has a wavelength much larger than the length of the unit cell. A proof of this assertion, together with comparisons of the stress required for the onset of buckling at the microscopic level to the stress required for the loss of ellipticity of the homogenized moduli for a two-dimensional layered medium under axial loading was first presented by TRIANTAFYLLIDIS and 
MAKER (1985). The advantage of considering the two-dimensional medium with only one set of fibers was that the problem was simple enough to permit an analytical solution for the microbuckling problem and an easy calculation of the homogenized moduli of the layered medium.

The relation between microscopic bifurcation and macroscopic loss of ellipticity described above was subsequently proven in general for arbitrary three-dimensional solids with periodic microstructures (including layered media as a subcase) by GFYMONAT et al. $(1990,1992)$. In contrast to the layered media case, homogenization calculations as well as bifurcation calculations for two- or three-dimensional periodic media whose unit cells are rectangular but not degenerate (i.e. with aspect ratios of the order of one) can only be done numerically. Some preliminary work in this direction by TRIANTAFYLLIDIS and MAKER (1987) has established the regions of microscopic stability (where microstructural buckling is excluded) and the regions of macroscopic stability (where shear bands in the homogenized material are excluded) for periodic hexagonal arrays of elastic media with circular second phase elastic inclusions under finite strain. The numerical determination of the regions of stability from the microstructural model is exceedingly time consuming which makes a parameter study of the problem very difficult. An analytically tractable model for a twodimensional composite with a nontrivial unit cell is therefore highly desirable, thus providing the motivation for the present work.

The present model for a fiber-reinforced composite which is described in Section 2 consists of a rectangular planar grillage whose beams are axially compressed in both directions. The matrix material of the composite is taken into account by its contribution to the average shear stiffness of the model. Using Bloch wave theory, one can find an analytical solution for the exact buckling problem of the model. The solution provides the lowest buckling load corresponding to a mode with given wavelengths in each direction. The calculation of the homogenized moduli of the model and the investigation of their ellipticity is described in Section 3. It is also shown there that the load level corresponding to the loss of ellipticity of the macroscopic homogenized moduli is always the same as the load level at the onset of the buckling eigenmode whose characteristic length is much larger than the unit cell length, in agreement with the general results in GeYmONAT et al. (1990,1992). Comparisons between the predictions for the onset of instability at the micro and macro levels for different types of models are given in Section 4. It is found that in the absence of a shear stiffness contribution from the matrix material the first instahility in the model can always be predicted from its macroscopic behavior, i.e. the critical mode is a global or long wavelength one. For adequately large values of the interstitial shear stiffness howcver, a local mode always precedes a global one, in which case the homogenized model overpredicts the stability region. The presentation is concluded by a discussion of results in Section 5 .

\section{OnSET OF THE First Instability in the Structure}

The planar grillage type model chosen as an idealization for a fiber reinforced composite permits the analytical calculation of the buckling loads and the homo- 
genized incremental moduli. Similar models have been presented in the structural mechanics literature pertaining to the study of stability for planar and space periodic lattice structures. WAH (1965) studied the stability of finite size rectangular gridworks for both in-plane and out-of-plane deformations. Unfortunately, the present stability results for infinite grillages in the absence of interstitial stiffness cannot be recovered from his work because the boundary effects in his models (due to their finite size) are absent in the present study.

Subsequent investigations on the buckling of periodic lattice structures were presented by Forman and HutChinson (1970), ANDERSON (1981) and ANDERSON and WILLIAMS (1986) for three-dimensional space frames, and by BAŽANT and CHRISTENSEN $(1972,1973)$ for planar frames. BAŽANT and CHRISTENSEN $(1972,1973)$, proposed essentially approximate continuum models with the goal of providing simpler continuum boundary value problems for the buckling of these structures as an alternative to (more difficult) exact frame formulations. All of these studies, motivated from structural applications for planar or space frames, naturally do not include the contribution of interstitial material stiffness - an important factor in the modeling of composites. Moreover, the aforementioned stability analyses were concerned with the study of finite size models. The proposed model and the issues it addresses are different enough from the ones existing in the literature to merit a separate presentation.

The two-dimensional periodic structure to be modeled here consists of an infinite planar frame, as shown in Fig. 1a. It is made up of horizontal and vertical beams welded together to form a rectangular grid pattern of unit cell dimensions $2 a_{1} \times 2 a_{2}$. The spaces between the beams are filled by an elastic material. Deformation of the frame is allowed only in the $x_{1}, x_{2}$ plane. This model is an idealization of a fiber reinforced composite where the beams represent the fibers and the interstitial elastic material represents the matrix.

All the beams in the $x_{x}$ direction have axial stiffness $E A_{x}$ and bending stiffness $E I_{x}$ with $E$ the beam material's Young's modulus, $A_{x}$ its cross-sectional area, and $I_{x}$ its in-plane moment of inertia. (From here on, Greek indices will always range from 1 to 2.) The interstitial material is homogeneous and linearly elastic, with thickness $t$, shear modulus $G$, and negligible normal moduli. The response of the structure is elastic. A small strain, moderate rotation theory is adopted to describe the behavior of the individual beams. The internal elastic energy of beam $J$ due to a deformation with axial displacement $u_{J}(x)$ and transverse displacement $v_{J}(x)$ is given by

$$
\mathscr{E}_{\text {bcam }_{l}}=\frac{1}{2} \int_{0}^{a_{I}}\left\{E A_{J}\left[\frac{\mathrm{d} u_{J}}{\mathrm{~d} x}+\frac{1}{2}\left(\frac{\mathrm{d} v_{J}}{\mathrm{~d} x}\right)^{2}\right]^{2}+E I_{J}\left[\frac{d^{2} v_{J}}{\mathrm{~d} x^{2}}\right]^{2}\right\} \mathrm{d} x .
$$

(From here on, capital Latin indices will always range from 1 to 4.) Note from Fig. 1a that $a_{3}=a_{1}, a_{4}=a_{2}, A_{3}=A_{1}, A_{4}=A_{2}, I_{3}=I_{1}$ and $I_{4}=I_{2}$.

The energy stored in the interstitial material of the unit cell is accounted for, in an approximate fashion, in terms of $t, G$ and the cell's average shear strain $\gamma$, namely,

$$
\mathscr{E}_{\text {interstitial }}=\frac{1}{2} G t \gamma^{2}\left(2 a_{1}\right)\left(2 a_{2}\right), \quad \gamma=\frac{U_{2}-U_{4}}{2 a_{2}}+\frac{V_{1}-V_{3}}{2 a_{1}}
$$

where the definition of the displacements $U_{i}, V_{i}$ and rotations $\theta_{i}$ of node $i$ may be seen from Fig. 1b. (From here on, lowercase Latin indices will always range from 0 to 4 .) 


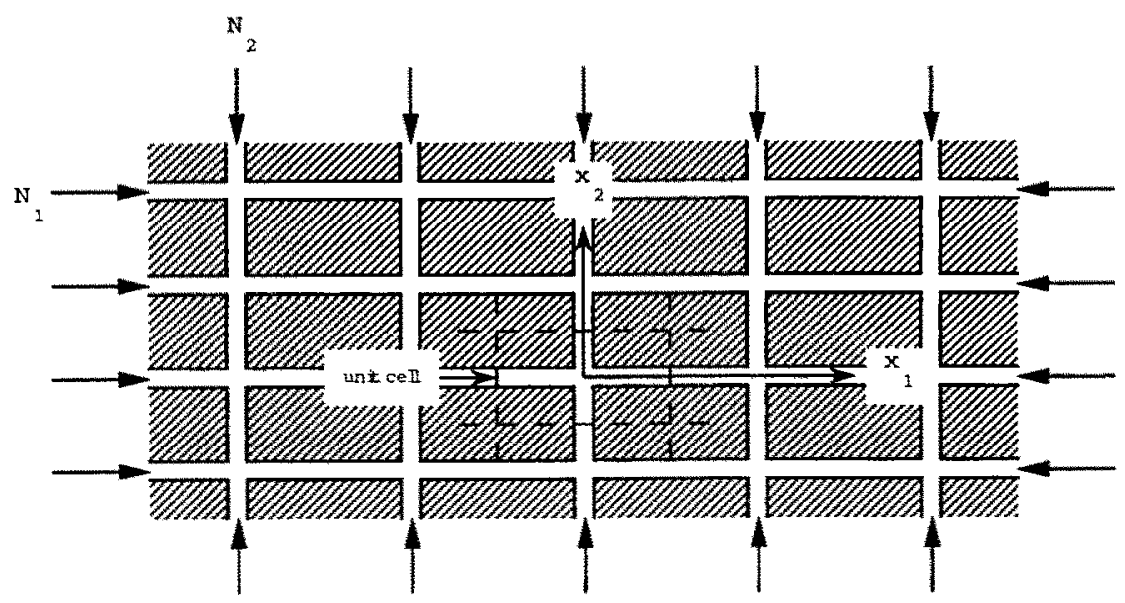

FIG. la. Schematic representation of the model.

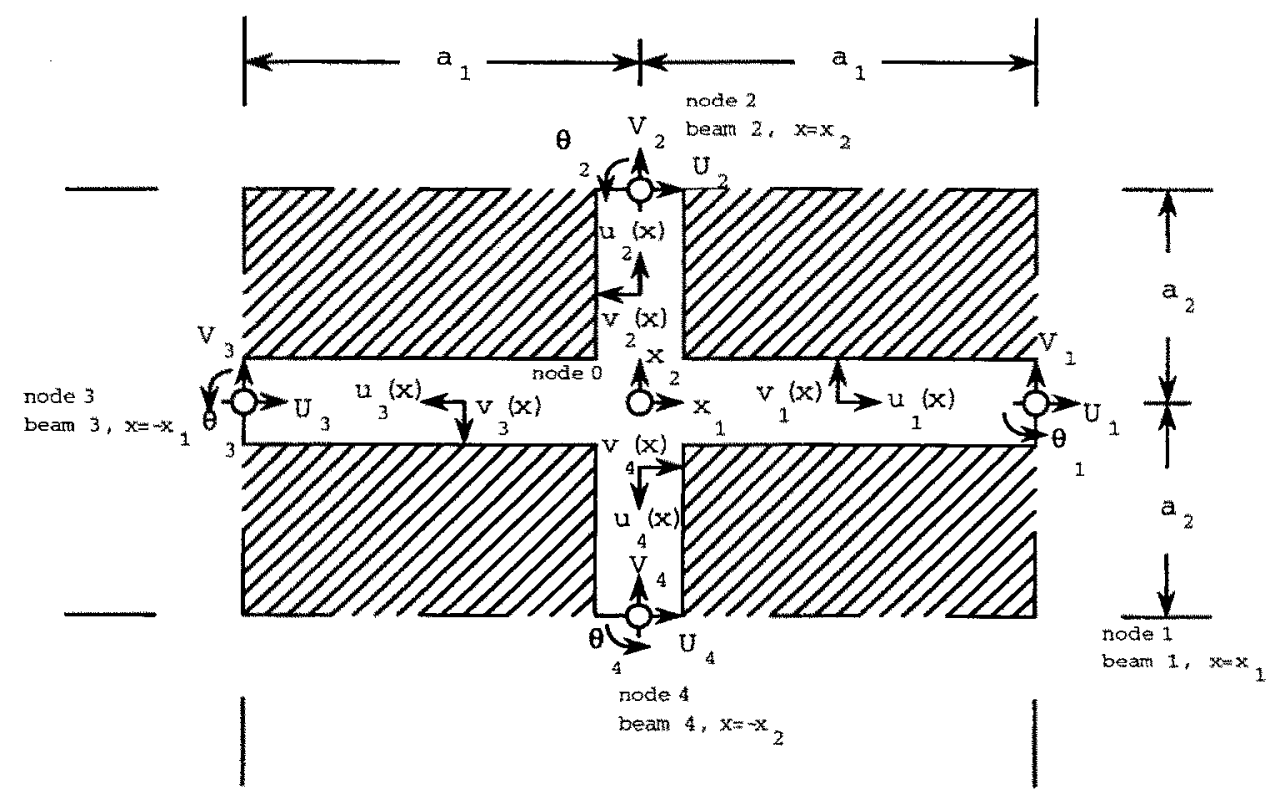

FlG. Ib. Unit cell. Definition of nodal displacements $U_{3}, V_{z}$ and $\theta_{i}$ in the global coordinate system and beam displacements $u_{j}$ and $v_{j}$ in the local coordinate system.

Compressive forces $N_{x}$, acting in the direction of the beams as indicated in Fig. la, are increasing in direct proportion to a scalar factor $\lambda$ which will be specified subsequently. The compressive normal stresses taken by the interstitial material are ignored, since the axial stiffness of the fibers is much larger than the normal moduli in the matrix material. Only the shear stresses in the interstitial material are considered. For $\lambda$ near zero, the beams develon internal axial forces, but no internal bending 
moments or shear resultants. Thus they remain straight, and the interstitial material is stress free, i.e. it has no overall shear strain. The corresponding unique and stable equilibrium solution is termed the principal solution.

As $\lambda$ keeps increasing away from zero, it reaches a particular value $\lambda_{\mathrm{c}}$ at which another solution, termed the buckled or bifurcated solution, emerges. Here, nonzero internal bending moments and shear resultants are developed in the beams. The structure's deformed configuration is now no longer rectangular nor (in general) periodic.

The critical load $\lambda_{\mathrm{c}}$ can be found by examining the solution of the rate (or incremental) form of the equilibrium equations. From here on, a quantity surmounted by a dot denotes the rate of change of the quantity with respect to the load parameter, i.e. $(\dot{)} \equiv \mathrm{d}() / \mathrm{d} \lambda$. At the onset of bifurcation, incremental equilibrium for the unit cell implies* the following relations between the force and displacement rate vectors $\hat{f}_{i}$ and $\dot{\mathbf{d}}_{i}$ at the five nodes of the unit cell:

$$
\begin{aligned}
& \dot{\mathbf{f}}_{0}=\left(\mathbf{K}_{11}^{1}+\mathbf{K}_{11}^{2}+\mathbf{K}_{11}^{3}+\mathbf{K}_{11}^{4}\right) \dot{\mathbf{d}}_{0}+\mathbf{K}_{12}^{1} \dot{\mathbf{d}}_{1}+\mathbf{K}_{12}^{2} \dot{\mathbf{d}}_{2}+\mathbf{K}_{12}^{3} \dot{\mathbf{d}}_{3}+\mathbf{K}_{12}^{4} \dot{\mathbf{d}}_{4}=\mathbf{0}, \\
& \dot{\mathbf{f}}_{1}=\mathbf{K}_{21}^{1} \dot{\mathbf{d}}_{0}+\left(\mathbf{K}_{22}^{1}+\mathbf{L}_{11}\right) \dot{\mathbf{d}}_{1}+\mathbf{L}_{12} \dot{\mathbf{d}}_{2}+\mathbf{L}_{13} \dot{\mathbf{d}}_{3}+\mathbf{L}_{14} \dot{\mathbf{d}}_{4}, \\
& \dot{\mathbf{f}}_{2}=\mathbf{K}_{21}^{2} \dot{\mathbf{d}}_{0}+\mathbf{L}_{21} \dot{\mathbf{d}}_{1}+\left(\mathbf{K}_{22}^{2}+\mathbf{L}_{22}\right) \dot{\mathbf{d}}_{2}+\mathbf{L}_{23} \dot{\mathbf{d}}_{3}+\mathbf{L}_{24} \dot{\mathbf{d}}_{4}, \\
& \dot{\mathbf{f}}_{3}=\mathbf{K}_{22}^{3} \dot{\mathbf{d}}_{0}+\mathbf{L}_{31} \dot{\mathbf{d}}_{1}+\mathbf{L}_{32} \dot{\mathbf{d}}_{2}+\left(\mathbf{K}_{22}^{3}+\mathbf{L}_{33}\right) \dot{\mathbf{d}}_{3}+\mathbf{L}_{34} \dot{\mathbf{d}}_{4}, \\
& \dot{\mathbf{f}}_{4}=\mathbf{K}_{21}^{4} \dot{\mathbf{d}}_{0}+\mathbf{L}_{41} \dot{\mathbf{d}}_{1}+\mathbf{I}_{42} \dot{\mathbf{d}}_{2}+\mathbf{L}_{43} \dot{\mathbf{d}}_{3}+\left(\mathbf{K}_{22}^{4}+\mathbf{L}_{44}\right) \dot{\mathbf{d}}_{4} .
\end{aligned}
$$

In the above equations, the elements of a particular nodal force vector $\mathbf{f}_{i}$ are the $x_{1}, x_{2}$ components of the nodal force plus the nodal moment about $x_{3}$. The elements of a particular displacement vector $\mathbf{d}_{i}$ are the work conjugate components of the corresponding elements of $\mathbf{f}_{i}$, namely the $x_{1}, x_{2}$ displacements of the node plus its rotation about $x_{3}$. In addition, $\mathbf{K}_{\alpha \beta}^{\prime}$ is the incremental stiffness matrix relating the $\alpha$ th elemental force rate vector to the $\beta$ th elemental displacement rate vector for beam $I$ (see Fig. lb). When the Greek index in the incremental stiffness matrix takes the value 1 , it corresponds to the beam end at the zero (center) node; 2 corresponds to the $I$ th (edge) node. Moreover $\mathbf{L}_{I J}$ is the incremental stiffness of the interstitial material alone, relating the $I$ th nodal force rate vector to the $J$ th nodal displacement rate vector. The calculation of $\mathbf{K}_{x \beta}^{i}$ for beams under axial loading follows a procedure exposed in LIVESLEY (1968). The calculation of $\mathbf{L}_{j,}$ from (2) is straightforward. For continuity in this presentation, as well as for reference purposes, the expressions for $\mathbf{K}_{x ; j}^{l}$ and $\mathbf{L}_{z j}$ are given in the Appendix, together with a brief outline of their derivation.

Recall that the infinite frame model under investigation is an approximation for a periodic, fiber-reinforced nonlinear elastic continuum. At bifurcation, the eigenmode for the continuum, $\dot{u}_{\alpha}\left(x_{1}, x_{2}\right)$, has to satisfy the incremental equilibrium equation, which is a homogeneous linear equation of the type $\left[L_{\alpha \beta ; \gamma \gamma}\left(x_{1}, x_{2}, \lambda_{\mathrm{c}}\right) \dot{u}_{;, \alpha}\right]_{, \beta}=0$, where $L_{x \beta ; \gamma}\left(x_{1}, x_{2}, \lambda_{\mathrm{c}}\right)$ is the continuum's incremental moduli tensor evaluated on the principal solution at the critical load. In view of the spatial periodicity of $L_{x / f / \gamma}$ [i.e. $L_{\alpha \beta \gamma ;}\left(x_{1}+2 m a_{1}, x_{2}+2 n a_{2}\right)=L_{x \beta ; \gamma ;}\left(x_{1}, x_{2}\right)$, for any integers $\left.m, n\right]$ it can be shown that

\footnotetext{
*The standard matrix method for nodal force equilibrium in frames is used; see for example LivesLfey (1968).
} 
the eigenmode is of the form $\dot{u}_{\alpha}\left(x_{1}, x_{2}\right)=\exp \left[\mathrm{i}\left(\omega_{1} x_{1}+\omega_{2} x_{2}\right)\right] p_{x}\left(x_{1}, x_{2}\right)$ where $p_{x}\left(x_{1}, x_{2}\right)$ is a periodic function of $x_{1}, x_{2}$ with the same spatial periodicity as $L_{\alpha \beta \gamma \delta}$ (see Geymonat et al., 1990, 1992). For the model considered here, one can deduce the following relation between $\dot{\mathbf{f}}_{i}$ and $\dot{\mathbf{d}}_{i}$ at bifurcation :

$$
\begin{array}{lll}
\dot{\mathbf{d}}_{1}=\mu_{1} \dot{\mathbf{d}}_{3}, & \dot{\mathbf{f}}_{1}=-\mu_{1} \dot{\mathbf{f}}_{3}, & \mu_{1} \equiv \exp \left(2 \mathrm{i}\left(\omega_{1} a_{1}\right),\right. \\
\dot{\mathbf{d}}_{2}=\mu_{2} \dot{\mathbf{d}}_{4}, & \dot{\mathbf{f}}_{2}=-\mu_{2} \dot{\mathbf{f}}_{4}, & \mu_{2} \equiv \exp \left(2 \mathrm{i} \omega_{2} a_{2}\right) .
\end{array}
$$

Introducing (4) into (3) and taking advantage of the identity (A.7) one obtains the following equivalent homogeneous system for $\dot{\mathbf{d}}_{0}, \dot{\mathbf{d}}_{3}, \dot{\mathbf{d}}_{4}$ :

$$
\begin{array}{r}
\left(\mathbf{K}_{11}^{1}+\mathbf{K}_{11}^{2}+\mathbf{K}_{11}^{3}+\mathbf{K}_{11}^{4}\right) \dot{\mathbf{d}}_{0}+\left(\mu_{1} \mathbf{K}_{12}^{1}+\mathbf{K}_{12}^{3}\right) \dot{\mathbf{d}}_{3}+\left(\mu_{2} \mathbf{K}_{12}^{2}+\mathbf{K}_{12}^{4}\right) \dot{\mathbf{d}}=\mathbf{0}, \\
\left(\bar{\mu}_{1} \mathbf{K}_{21}^{1}+\mathbf{K}_{21}^{3}\right) \dot{\mathbf{d}}_{0}+\left(\mathbf{K}_{22}^{1}+\mathbf{K}_{22}^{3}+\left[2-\mu_{1}-\bar{\mu}_{1}\right] \mathbf{L}_{11}\right) \dot{\mathbf{d}}_{3}+\left(\left[1-\bar{\mu}_{1}\right]\left[1-\mu_{2}\right] \mathbf{L}_{12}\right) \dot{\mathbf{d}}_{4}=\mathbf{0}, \\
\left(\bar{\mu}_{2} \mathbf{K}_{21}^{2}+\mathbf{K}_{21}^{4}\right) \dot{\mathbf{d}}_{0}+\left(\left[1-\mu_{1}\right]\left[1-\bar{\mu}_{2}\right] \mathbf{L}_{21}\right) \dot{\mathbf{d}}_{3} \\
+\left(\mathbf{K}_{22}^{2}+\mathbf{K}_{22}^{4}+\left[2-\mu_{2}-\bar{\mu}_{2}\right] \mathbf{L}_{22}\right) \dot{\mathbf{d}}_{4}=\mathbf{0} .
\end{array}
$$

As usual, $\bar{\mu}_{x}$ denotes the complex conjugate of $\mu_{\alpha}$. The second and third relations of (5), linear in $\dot{\mathbf{d}}_{i}$, are used to solve for $\dot{\mathbf{d}}_{3}$ and $\dot{\mathbf{d}}_{4}$ in terms of $\dot{\mathbf{d}}_{0}$. The results are then applied to the first relation of (5) to obtain

$$
\begin{aligned}
\mathbf{K}[\lambda] \dot{\mathbf{d}}_{0}= & \mathbf{0}, \\
\mathbf{K}[\lambda] \equiv & \mathbf{K}_{11}^{1}+\mathbf{K}_{11}^{2}+\mathbf{K}_{11}^{3}+\mathbf{K}_{11}^{4}-\left(\mu_{1} \mathbf{K}_{12}^{1}+\mathbf{K}_{12}^{3}\right) \mathbf{M}_{11}\left(\bar{\mu}_{1} \mathbf{K}_{21}^{1}+\mathbf{K}_{21}^{3}\right) \\
& -\left(\mu_{1} \mathbf{K}_{12}^{1}+\mathbf{K}_{12}^{3}\right) \mathbf{M}_{12}\left(\bar{\mu}_{2} \mathbf{K}_{21}^{2}+\mathbf{K}_{21}^{4}\right)-\left(\mu_{2} \mathbf{K}_{12}^{2}+\mathbf{K}_{12}^{4}\right) \mathbf{M}_{21} \\
& \times\left(\bar{\mu}_{1} \mathbf{K}_{21}^{1}+\mathbf{K}_{21}^{3}\right)-\left(\mu_{2} \mathbf{K}_{12}^{2}+\mathbf{K}_{12}^{4}\right) \mathbf{M}_{22}\left(\bar{\mu}_{2} \mathbf{K}_{21}^{2}+\mathbf{K}_{21}^{4}\right), \\
\mathbf{M}_{11} \equiv & \left\{\mathbf{K}_{22}^{1}+\mathbf{K}_{22}^{3}+\left[2-\mu_{1}-\bar{\mu}_{1}\right] \mathbf{L}_{11}-\left[2-\mu_{1}-\bar{\mu}_{1}\right]\left[2-\mu_{2}-\bar{\mu}_{2}\right] \mathbf{L}_{12}\right. \\
& \left.\times\left(\mathbf{K}_{22}^{2}+\mathbf{K}_{22}^{1}+\left[2-\mu_{2}-\bar{\mu}_{2}\right] \mathbf{L}_{22}\right)^{-1} \mathbf{L}_{21}\right\}^{-1}, \\
\mathbf{M}_{12} \equiv- & {\left[1-\bar{\mu}_{1}\right]\left[1-\mu_{2}\right] \mathbf{M}_{11} \mathbf{L}_{12}\left(\mathbf{K}_{22}^{2}+\mathbf{K}_{22}^{4}+\left[2-\mu_{2}-\bar{\mu}_{2}\right] \mathbf{L}_{22}\right)^{-1}, } \\
\mathbf{M}_{21} \equiv- & {\left[1-\mu_{1}\right]\left[1-\bar{\mu}_{2}\right] \mathbf{M}_{22} \mathbf{L}_{21}\left(\mathbf{K}_{22}^{1}+\mathbf{K}_{22}^{3}+\left[2-\mu_{1}-\bar{\mu}_{1}\right] \mathbf{L}_{11}\right)^{-1}, } \\
\mathbf{M}_{22} \equiv\{ & \left\{\mathbf{K}_{22}^{2}+\mathbf{K}_{22}^{4}+\left[2-\mu_{2}-\bar{\mu}_{2}\right] \mathbf{L}_{22}-\left[2-\mu_{1}-\bar{\mu}_{1}\right]\left[2-\mu_{2}-\bar{\mu}_{2}\right] \mathbf{L}_{21}\right. \\
& \left.\times\left(\mathbf{K}_{22}^{1}+\mathbf{K}_{22}^{3}+\left[2-\mu_{1}-\bar{\mu}_{1}\right] \mathbf{L}_{11}\right)^{-1} \mathbf{L}_{12}\right\}^{-1} .
\end{aligned}
$$

Al bifurcation $\left(\lambda=\lambda_{\mathrm{c}}\right)$ a nontrivial solution to (6) exists when

$$
\operatorname{Det}\left(\mathbf{K}\left[\lambda_{\mathrm{c}}\right]\right)=0
$$

The expressions for $\mathbf{K}_{\alpha \beta}^{l}$ and $\mathbf{L}_{I J}$ from the Appendix [see (A.4) and (A.7)] are substituted into (6). After carrying out the required matrix inversions and multiplications, the components of the $3 \times 3$ stability matrix $\mathbf{K}[\lambda]$ are found to be

$$
\begin{aligned}
K_{11}\left[\lambda, s_{1}, s_{2}\right]= & E\left\{2\left[\left(a_{1}\left(r_{1}\right)^{2} s_{1} / c_{1}\right)+\left(a_{2}\left(r_{2}\right)^{4} s_{2} / c_{2}\right)\left(F_{2}[\lambda]-B_{2}[\lambda]\right)\right]\right. \\
& \left.+4\left[s_{2}\left(1-s_{2}\right) a_{1} / a_{2}\right] / D\left[\lambda, s_{1}, s_{2}\right]\right\},
\end{aligned}
$$




$$
\begin{aligned}
K_{12}\left[\lambda, s_{1}, s_{2}\right]= & E\left\{4\left[s_{1}\left(1-s_{1}\right) s_{2}\left(1-s_{2}\right)\right]^{1 / 2} / D\left[\lambda, s_{1}, s_{2}\right]\right\} \\
= & K_{21}\left[\lambda, s_{1}, s_{2}\right], \\
K_{13}\left[\lambda, s_{1}, s_{2}\right]= & -\mathrm{i} E\left[s_{2}\left(1-s_{2}\right)\right]^{1 / 2}\left\{\left(2\left(a_{2}\right)^{2}\left(r_{2}\right)^{4} / c_{2}\right) F_{2}[\lambda]\right. \\
& \left.+2 a_{1} N\left[\lambda, s_{1} s_{2}\right] / D\left[\lambda, s_{1}, s_{2}\right]\right\} \\
= & -K_{31}\left[\lambda, s_{1}, s_{2}\right], \\
K_{22}\left[\lambda, s_{1}, s_{2}\right]= & E\left\{2\left[\left(a_{2}\left(r_{2}\right)^{2} s_{2} / c_{2}\right)+\left(a_{1}\left(r_{1}\right)^{4} s_{1} / c_{1}\right)\left(F_{1}[\lambda]-B_{1}[\lambda]\right)\right]\right. \\
& \left.+4\left[s_{1}\left(1-s_{1}\right) a_{2} / a_{1}\right] / D\left[\lambda, s_{1}, s_{2}\right]\right\}, \\
K_{23}\left[\lambda, s_{1}, s_{2}\right]= & i E\left[s_{1}\left(1-s_{1}\right)\right]^{1 / 2}\left\{\left(2\left(a_{1}\right)^{2}\left(r_{1}\right)^{4} / c_{1}\right) F_{1}[\lambda]\right. \\
& \left.-2 a_{2} N\left[\lambda, s_{1}, s_{2}\right] / D\left[\lambda, s_{1}, s_{2}\right]\right\} \\
= & -K_{32}\left[\lambda, s_{1}, s_{2}\right], \\
& +\left(2\left(a_{2}\right)^{3}\left(r_{2}\right)^{4} / c_{2}\right)\left(F_{2}[\lambda]-s_{2} G_{2}[\lambda]\right) \\
& \left.+a_{1} a_{2}\left(N\left[\lambda, s_{1}, s_{2}\right]\right)^{2} / D\left[\lambda, s_{1}, s_{2}\right]\right\} .
\end{aligned}
$$

The new terms introduced for convenience in the above equations are as follows:

$$
\begin{aligned}
s_{\alpha} \equiv & \left(\sin \left[\omega_{\alpha} a_{\alpha}\right]\right)^{2}, \quad c_{\alpha} \equiv 12 I_{\alpha} /\left(A_{\alpha}\right)^{2}, \quad r_{\alpha} \equiv\left(12 I_{\alpha} / A_{\alpha}\right)^{1 / 2} / a_{\alpha}, \\
B_{\alpha}[\lambda] \equiv & \left(\beta_{\alpha}[\lambda]\right)^{2} / 12 \equiv c_{\alpha} N_{\alpha}[\lambda] /\left(\left(a_{\alpha}\right)^{2} E\left(r_{\alpha}\right)^{4}\right), \\
F_{\alpha}[\lambda] \equiv & \left(\left(\sigma_{\alpha}[\lambda]\right)^{2}-\left(\tau_{x}[\lambda]\right)^{2}\right) /\left(12\left(\sigma_{\alpha}[\lambda]\right)^{2}\right), \\
G_{\alpha}[\lambda] \equiv & \left\{\left(\sigma_{\alpha}[\lambda]+\tau_{\alpha}[\lambda]\right)^{2} /\left[2\left(\sigma_{\alpha}[\lambda]+\tau_{\alpha}[\lambda]\right)-\left(\beta_{\alpha}[\lambda]\right)^{2}\right]\right. \\
& \left.-\left(\tau_{\alpha}[\lambda]\right)^{2} / \sigma_{\alpha}[\lambda]\right\} / 12 \\
N\left[\lambda, s_{1}, s_{2}\right] \equiv & s_{1} \phi_{1}[\hat{\lambda}] / \phi_{1}[\lambda]-s_{2} \phi_{2}[\lambda] / \phi_{2}[\lambda], \\
D\left[\lambda, s_{1}, s_{2}\right] \equiv & (E /(G t))+2 a_{2} c_{1} s_{1} /\left(\left(a_{1}\right)^{2}\left(r_{1}\right)^{4} \phi_{1}[\lambda]\right) \\
& +2 a_{1} c_{2} s_{2} /\left(\left(a_{2}\right)^{2}\left(r_{2}\right)^{4} \phi_{2}[\lambda]\right),
\end{aligned}
$$

where the functions $\sigma_{\alpha}\left[\beta_{\alpha}\right]$ and $\tau_{x}\left[\beta_{\alpha}\right]\left(\beta_{\alpha}=\beta_{\alpha}[\lambda]\right)$ as well as $\phi_{\alpha}\left[\beta_{\alpha}\right]$ and ${ }_{\phi_{x}}^{2}\left[\beta_{\alpha}\right]$ are given in the Appendix [sec (A.3)]. The dependence of the $N_{x}[\lambda]$ (the axial forces on the beams) on the load parameter $\lambda$ will be described in the next section.

Notice that the stability matrix $\mathbf{K}$ is a function only of the load parameter $\lambda$ and the $s_{x}$ terms, for a particular planar frame model with given geometry and material properties. Thus from (7), Det $\left(\mathbf{K}\left[\lambda, s_{1}, s_{2}\right]\right)=0$, one can find the value of the load parameter at the onset of bifurcation implicitly as a function of $s_{1}, s_{2}$, i.e. $\hat{\lambda}\left[s_{1}, s_{2}\right]$. Assuming that the load parameter is always positive, of interest are the lowest roots $\hat{\lambda}\left[s_{1}, s_{2}\right]$ of $\operatorname{Det}\left(\mathbf{K}\left[\hat{\lambda}, s_{1}, s_{2}\right]\right)=0$.

For each pair $\left(s_{1}, s_{2}\right) \neq(0,0)$, the value of $\hat{\lambda}\left[s_{1}, s_{2}\right]$ is found numerically using a 
straightforward incremental procedure which detects the first change in the sign of Det $(\mathbf{K}[\lambda])$ from positive* to negative. Depending on the size of $\Delta \lambda$ (the increment in 2) a simple bisection method can also be used for more accuracy in the calculation of $\hat{\lambda}$. The case of $s_{1}=s_{2}=0$ is treated in the same manner, except Det $\left(\mathbf{K}\left[\hat{\lambda}, s_{1}, s_{2}\right]\right)$ is replaced by $K_{33}[\lambda, 0,0]$, as will be explained subsequently.

For a given geometry of the model, results are obtained in the form of surfaces $\hat{\lambda}\left(s_{1}, s_{2}\right)$ where $0 \leqslant s_{1} \leqslant 1,0 \leqslant s_{2} \leqslant 1$. The wanted critical load $\lambda_{\mathrm{c}}$ is the lowest point of that surface, i.e.

$$
\begin{aligned}
& \lambda_{\mathrm{c}}=\underset{\min \left[\hat{\lambda}\left(s_{1}, s_{2}\right)\right] ;}{0 \leqslant s_{i} \leqslant 1,0 \leqslant s_{2} \leqslant 1} ;\left\{\begin{array}{ll}
\left(s_{1}, s_{2}\right) \neq(0,0) & \\
\operatorname{Det}\left(\mathbf{K}\left[\lambda, s_{1}, s_{2}\right]\right)>0, & 0 \leqslant \lambda<\hat{\lambda} \\
\operatorname{Det}\left(\mathbf{K}\left[\lambda, s_{1}, s_{2}\right]\right)=0, & \lambda=\hat{\lambda}
\end{array}\right\} \\
& \text { or }\left\{\begin{array}{ll}
\left(s_{1}, s_{2}\right)=(0,0) & \\
K_{33}\left[\lambda, s_{1}, s_{2}\right]>0, & 0 \leqslant \lambda<\hat{\lambda} \\
K_{33}\left[\lambda, s_{1}, s_{2}\right]=0, & \lambda=\hat{\lambda}
\end{array}\right\} .
\end{aligned}
$$

For all of the structures investigated, $\hat{\lambda}\left[s_{1}, s_{2}\right]$ is a continuous smooth function of its arguments except at the origin, $\left(s_{1}, s_{2}\right)=(0,0)$. Inspection of (8) identifies the mathematical reason for this. When $s_{1}=s_{2}=0$, all the components of $\mathbf{K}$ except for $K_{33}$ are identically equal to zero. Thus $\operatorname{Det}(\mathbf{K})$ is identically zero for any value of the load parameter. The physical reason for the singularity has to do with the fact that the $\left(s_{1}, s_{2}\right)=(0,0)$ point corresponds to two different families of bifurcation eigenmodes of the structurc.

The first family consists of all modes where each mode's wavelength $\left(L_{x}\right)$ fits into the length of the unit cell an integral number of times $\left(2 a_{\alpha}=k_{\alpha} L_{\alpha}: k_{\alpha} \in\{\mathbb{N}\}\right)$. These are termed periodic bifurcation modes and can be seen to result in $s_{x}=\left(\sin \left[\omega_{x} a_{x}\right]\right)^{2}=0$ upon substitution of the relation between wavelength and angular frequency $\left(L_{\alpha}=2 \pi / \omega_{\alpha}\right)$ into the above definition to obtain $2 a_{\alpha} \omega_{x}=2 \pi k_{x}$. Hence from (8) $K_{33}$ is the only component of $\mathbf{K}$ to be non-zero for the case $s_{1}=s_{2}=0$. Excluding translational rigid body modes, one finds the load parameter $\hat{\lambda}[0,0]$ corresponding to the periodic bifurcation modes via $K_{33}[\lambda, 0,0]=\left(\left(a_{1}\right)^{3}\left(r_{1}\right)^{4} / c_{1}\right) F_{1}[\lambda]+$ $\left(\left(a_{2}\right)^{3}\left(r_{2}\right)^{4} / c_{2}\right) F_{2}[\lambda]=0$ in the manner described in $(10)$.

The second family of modes corresponding to the point $\left(s_{1}, s_{2}\right)=(0,0)$ consists of all modes with wavelengths much greater than the unit cell size $\left(L_{\alpha}=2 \pi / \omega_{\alpha} \gg a_{\alpha}\right)$ and thus $\omega_{\alpha} a_{x} \rightarrow 0$. These are termed long wavelength modes and are completely different from the periodic modes because their displacement fields vary very slowly with position. The presence of two significantly different families of modes at the neighborhood of $\left(s_{1}, s_{2}\right)=(0,0)$ explains the existence of the singularity at the point on the $\hat{\lambda}\left[s_{1}, s_{2}\right]$ surface.

The bifurcation loads corresponding to the long wavelength modes are found as follows. First, define the terms $\varepsilon$ and $n_{\alpha}$ :

\footnotetext{
* Note: from (8) one can show that $\mathbf{K}[\lambda=0]$ is a positive definite Hermitian $\left(\overline{\mathbf{K}}=\mathbf{K}^{\mathrm{T}}\right)$ matrix and thus $\operatorname{Det}(\mathbf{K}[i=0])>0$
} 


$$
\omega_{x} \equiv \varepsilon n_{\alpha}, \quad n_{1}^{2}+n_{2}^{2} \equiv 1 \Rightarrow \varepsilon^{2}=\omega_{1}^{2}+\omega_{2}^{2} \text {. }
$$

In view of the series expansion $\sin \left(\omega_{\alpha} a_{\alpha}\right) \simeq \omega_{\alpha} a_{\alpha}-\left(\omega_{\alpha} a_{\alpha}\right)^{3} / 3 !+\cdots$, introduction of (11) into (8) provides the following result upon evaluating the determinant of the stability matrix $\mathbf{K}$ :

$$
\begin{aligned}
\operatorname{Det}\left(\mathbf{K}\left[\lambda_{,}, s_{1}, s_{2}\right]\right)= & \varepsilon^{4}\left(A_{11}[\lambda] n_{1}^{4}+A_{12}[\lambda] n_{1}^{4} n_{2}^{4}+A_{22}[\lambda] n_{2}^{4}\right)+\mathcal{O}\left(\varepsilon^{6}\right) \\
A_{11}[\lambda] \equiv & 8 E^{3}\left\{( a _ { 1 } ^ { 2 } r _ { 1 } ^ { 6 } / c _ { 1 } ^ { 2 } ) \left[-\left(a_{1}^{3} r_{1}^{4} / c_{1}\right) F_{1}[\lambda] B_{1}[\lambda]\right.\right. \\
& \left.+\left(a_{2}^{3} r_{2}^{4} / c_{2}\right) F_{2}[\lambda]\left(F_{1}[\lambda]-B_{1}[\lambda]\right)\right]+2(G t / E)\left(a_{2} r_{1}^{2} / c_{1}\right) \\
& \left.\times\left(\left[a_{1}^{3} r_{1}^{4} / c_{1}\right] F_{1}[\lambda]+\left[a_{2}^{3} r_{2}^{4} / c_{2}\right] F_{2}[\lambda]\right)\right\} a_{1}^{4}, \\
A_{22}[\lambda] \equiv & 8 E^{3}\left\{( a _ { 2 } ^ { 2 } r _ { 2 } ^ { 6 } / c _ { 2 } ^ { 2 } ) \left[-\left(a_{2}^{3} r_{2}^{4} / c_{2}\right) F_{2}[\lambda] B_{2}[\lambda]\right.\right. \\
& \left.+\left(a_{1}^{3} r_{1}^{4} / c_{1}\right) F_{1}[\lambda]\left(F_{2}[\lambda]-B_{2}[\lambda]\right)\right]+2(G t / E)\left(a_{1} r_{2}^{2} / c_{2}\right) \\
& \left.\times\left(\left[a_{2}^{3} r_{2}^{4} / c_{2}\right] F_{1}[\lambda]+\left[a_{1}^{3} r_{1}^{4} / c_{1}\right] F_{2}[\lambda]\right)\right\} a_{2}^{4}, \\
A_{12}[\lambda]= & 8 E^{3}\left\{[ a _ { 1 } a _ { 2 } r _ { 1 } ^ { 2 } r _ { 2 } ^ { 2 } / ( c _ { 1 } c _ { 2 } ) ] \left(\left[a_{1}^{3} r_{1}^{4} / c_{1}\right] F_{1}[\lambda]\right.\right. \\
& \left.+\left[a_{2}^{3} r_{2}^{4} / c_{2}\right] F_{2}[\lambda]\right)-\left[a_{1} a_{2} r_{1}^{4} r_{2}^{4} /\left(c_{1} c_{2}\right)\right] \\
& \times\left[\left(a_{1}^{3} r_{1}^{4} / c_{1}\right) F_{1}[\lambda] B_{1}[\lambda]\left(F_{2}[\lambda]-B_{2}[\lambda]\right)\right. \\
& \left.+\left(a_{2}^{3} r_{2}^{4} / c_{2}\right) F_{2}[\lambda] B_{2}[\lambda]\left(F_{1}[\lambda]-B_{1}[\lambda]\right)\right] \\
& -2(G t / E)\left[1 /\left(a_{1} a_{2}\right)\right]\left(\left[a_{1}^{3} r_{1}^{4} / c_{1}\right] F_{1}[\lambda]\right. \\
& \left.+\left[a_{2}^{3} r_{2}^{4} / c_{2}\right] F_{2}[\lambda]\right)\left(\left[a_{1}^{3} r_{1}^{4} / c_{1}\right] B_{1}[\lambda]\right. \\
& \left.\left.+\left[a_{2}^{3} r_{2}^{4} / c_{2}\right] B_{2}[\lambda]\right)\right\} a_{1}^{2} a_{2}^{2} .
\end{aligned}
$$

From (12) it is clear that for very small $\varepsilon$ the only significant term in $\operatorname{Det}\left(\mathbf{K}\left[\lambda, s_{1}, s_{2}\right]\right)$ is the biquadratic polynomial $A_{11}[\lambda] n_{1}^{4}+A_{12}[\lambda] n_{1}^{4} n_{2}^{4}+A_{22}[\lambda] n_{2}^{4}$. Thus the load parameters corresponding to the long wavelength bifurcation modes for a particular model vary only with the unit vector $\left(n_{1}, n_{2}\right)$.

$$
\begin{aligned}
\lim _{x \rightarrow 0} \hat{\lambda}\left[\left(\varepsilon n_{1} a_{1}\right)^{2},\left(c n_{2} a_{2}\right)^{2}\right]=\hat{\Lambda}\left(n_{1}, n_{2}\right) ; \\
\left.\qquad \begin{array}{l}
A_{11}[\lambda] n_{1}^{4}+A_{12}[\lambda] n_{1}^{4} n_{2}^{4}+A_{22}[\lambda] n_{2}^{4}>0, \quad 0 \leqslant \lambda<\hat{\Lambda} \\
A_{11}[\hat{\lambda}] n_{1}^{4}+A_{12}[\lambda] n_{1}^{4} n_{2}^{4}+A_{22}[\lambda] n_{2}^{4}=0, \quad \lambda=\hat{\Lambda}
\end{array}\right\} .
\end{aligned}
$$

Of all the values of $\hat{\Lambda}$, of interest is $\Lambda_{c}$, the lowest load parameter corresponding to the long wavelength bifurcation modes, i.e. the minimum of $\hat{\Lambda}$ over all possible unit vectors $\left(n_{1}, n_{2}\right)$. From (13), it is found to be*

$$
\begin{aligned}
& \Lambda_{c}=\min \left[\Lambda_{1}, \Lambda_{2}, \Lambda_{\prime}\right] ; \quad\left\{\begin{array}{cc}
A_{\alpha x}[\lambda]>0, & 0 \leqslant \lambda<\Lambda_{\alpha} \\
A_{\alpha x}[\lambda]=0, & \lambda=\Lambda_{\alpha}
\end{array}\right\} \\
& \text { and }\left\{\begin{array}{l}
\mathscr{D}[\lambda] \equiv 4 A_{11}[\lambda] A_{22}[\lambda]-A_{12}^{2}[\lambda] \\
\mathscr{D}[\lambda]>0, \quad 0 \leqslant \lambda<\Lambda_{1} \\
\mathscr{D}[\lambda]=0, \quad \lambda=\Lambda_{1}
\end{array}\right\} \text {. }
\end{aligned}
$$

*A solution for $\Lambda$ does not necessarily exist for every model 
For $\Lambda_{\mathrm{c}}=\Lambda_{1},\left(n_{1}\right)_{\mathrm{c}}=1, \quad\left(n_{2}\right)_{\mathrm{c}}=0$; for $\Lambda_{\mathrm{c}}=\Lambda_{2},\left(n_{1}\right)_{\mathrm{c}}=0,\left(n_{2}\right)_{\mathrm{c}}=1$; and for $\Lambda_{\mathrm{c}}=\Lambda_{2 x},\left(n_{1} / n_{2}\right)_{\mathrm{c}}=\left[-A_{12} /\left(2 A_{11}\right)\right]^{1 / 2}=\left[-2 A_{22} / A_{12}\right]^{1 / 2}$. The physical interpretation of the above three possibilities will be given in the next section where the long wavelength bifurcation result $\hat{\Lambda}$ will be derived from considerations involving the macroscopic (homogenized) properties of the structure.

\section{Stability of the. Homogfnitfid Structurf.}

In the previous section, (10) solves the exact (microscopic) instability problem for the onset of the first bifurcation in the infinite periodic structure model, because it places no restrictions on the eigenmodes investigated. Of interest in this section is the averaged (macroscopic) behavior of the model and a study of its stability. The periodic structure's macroscopic behavior is characterized by the homogenized incremental moduli of the structure which relate the average stress increment to the average strain increment where the average is taken over a unit cell which is the characteristic volume for the periodic material. The stability of the homogenized material is reflected in the ellipticity of the thus derived spatially constant homogenized incremental moduli; loss of ellipticity in a homogeneous material with spatially constant properties means that the homogeneous stress state in a Dirichlet (displacement controlled) problem is no longer an energy minimizer and is thus unstable. For a readable account of the relations between material stability and corresponding constitutive restrictions see BALL (1977) for elastic materials and RICE (1976) for elastoplastic ones as well as references quoted therein.

Following BENSOUSSAN et al. (1978), the homogenized incremental moduli $\mathscr{L}_{\alpha \beta \gamma ;}[\lambda]$ of a periodic medium can be found explicitly from the values of the incremental moduli $L_{x p ; i ;}\left[x_{1}, x_{2}, \lambda\right]$ of the unit cell $Y$. To accomplish this, one must determine the incremental response of the unit cell to an applied average incremental deformation $\delta_{x k} \delta_{\beta i}$. More specifically,

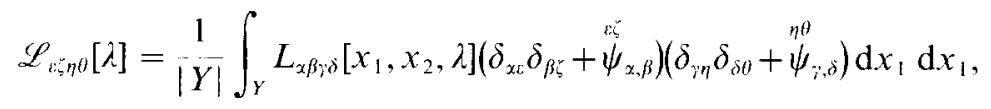

where $\psi_{x}\left[x_{1}, x_{2}\right]$ are $Y$ periodic functions in the unit cell and $|Y|$ denotes the volume of the unit cell. Note that a function $\psi_{\alpha}\left[x_{1}, x_{2}\right]$ is termed $Y$ periodic if $\psi_{\alpha}$ assumes the same values at opposite faces of the unit cell. All of the higher derivatives of $\psi_{\alpha}$ (or appropriate jump conditions where derivatives fail to exist) must also have that property. To summarize,

$$
\begin{array}{ll}
\psi_{x}\left(x_{1},-a_{2}\right)=\psi_{x}\left(x_{1}, a_{2}\right), & \psi_{\alpha, \beta}\left(x_{1},-a_{2}\right)=\psi_{\alpha, \beta}\left(x_{1}, a_{2}\right), \ldots \\
\psi_{x}\left(-a_{1}, x_{2}\right)=\psi_{x}\left(a_{1}, x_{2}\right), & \psi_{\alpha, \beta}\left(-a_{1}, x_{2}\right)=\psi_{\alpha, \beta}\left(a_{1}, x_{2}\right), \ldots
\end{array}
$$

The $\psi_{x}$ are found from the following variational statement which holds for all $Y$ periodic functions $\delta \psi_{\gamma}$ :

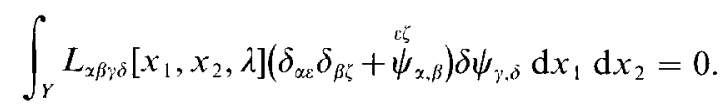


In view of the existence of a strain energy $\mathscr{E}$ per unit cell of the structure where $\mathscr{E}=\mathscr{E}_{\text {interstitial }}+\sum_{J=1}^{4} \mathscr{E}_{\text {heam, }}$ [see (1) and (2)],

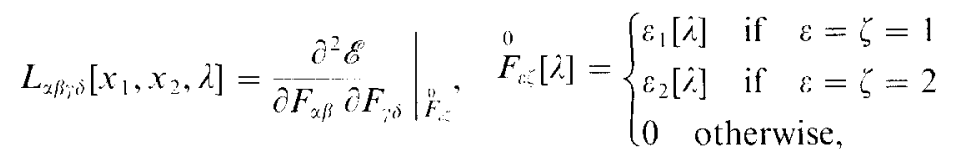

where $\varepsilon_{1}$ and $\varepsilon_{2}$ are the strains in the $x_{1}$ and $x_{2}$ directions respectively in the principal solution.

Substitution of (1), (2) and (18) into (15) provides the following expressions for the homogenized incremental moduli :

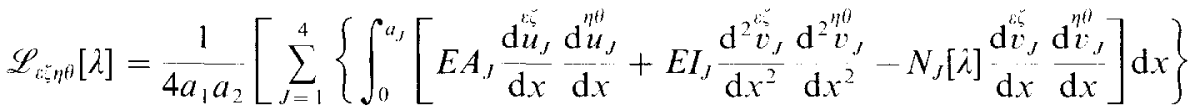

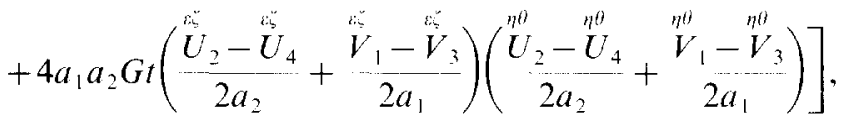

where ${ }^{n \zeta} u_{J}(x)$ and ${ }_{v_{J}}(x)$ denote the horizontal and vertical components of the displacement in the local coordinate system of member $J$ corresponding to the field $\delta_{x:} x_{\xi}+\psi_{x}\left(x_{1}, x_{2}\right)$, i.e. $\stackrel{\varepsilon}{u}_{1}(x)=x \delta_{1 \xi} \delta_{1 \zeta}+\psi_{1}(x, 0), \quad v_{1}(x)=x \delta_{2 \xi} \delta_{1 \xi}+\psi_{2}(x, 0)$, $\ddot{u}_{2}(x)=x \delta_{2 x} \delta_{2 \zeta}+\psi_{2}(0, x),-v_{2}(x)=x \delta_{1 k} \delta_{2 \zeta}+\psi_{1}(0, x)$, etc. In addition $U_{i}$ and $V_{1}$ denote the horizontal and vertical components of the displacement at node $i$ corresponding to the same field, i.e. $U_{1}=a_{1} \delta_{1 \varepsilon} \delta_{1 \xi}+\psi_{1}\left(a_{1}, 0\right), V_{1}=a_{1} \delta_{2 \varepsilon} \delta_{1 \xi}+\psi_{2}\left(a_{1}, 0\right)$, $U_{2}=a_{2} \delta_{1 \xi} \delta_{2 \zeta}+\psi_{1}\left(0, a_{2}\right), V_{2}=a_{2} \delta_{2 \xi} \delta_{2 \xi}+\psi_{2}\left(0, a_{2}\right)$, etc. as seen in Fig. $1 \mathrm{~b}$. ${ }^{*}$

Substitution of (1), (2) and (15) into (17) provides the following variational statement defining the terms $u_{J}(x)$ and $v_{J}(x)$ :

$$
\begin{aligned}
\sum_{J=1}^{4}\left\{\int_{0}^{a_{J}}\left[E A_{J} \frac{\mathrm{d} u_{J}}{\mathrm{~d} x} \frac{\mathrm{d} \delta u_{J}}{\mathrm{~d} x}+E I_{J} \frac{\mathrm{d}^{2} v_{J}}{\mathrm{~d} x^{2}} \frac{\mathrm{d}^{2} \delta v_{J}}{\mathrm{~d} x^{2}}-N_{J}[\lambda] \frac{\mathrm{d} v_{J}}{\mathrm{~d} x} \frac{\mathrm{d} \delta v_{J}}{\mathrm{~d} x}\right] \mathrm{d} x\right\} \\
+4 a_{1} a_{2} G t\left(\frac{U_{2}-U_{4}}{2 a_{2}}+\frac{V_{1}-V_{3}}{2 a_{1}}\right)\left(\frac{\delta U_{2}-\delta U_{4}}{2 a_{2}}+\frac{\delta V_{1}-\delta V_{3}}{2 a_{1}}\right)-0 .
\end{aligned}
$$

The derivations of the Euler-Lagrange differential equations and boundary conditions for $u_{J}(x)$ and $v_{J}(x)$ are straightforward. The differential equations are obtained via integration by parts and are the familiar ones of the beam buckling problem:

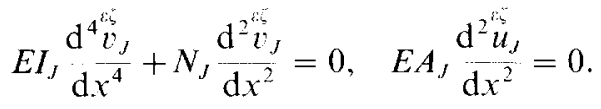

\footnotetext{
* Note that $u_{J}(x)$ and $v_{J}(x)$ are expressed in the local coordinate system of member $J$, while $\psi_{x}\left(x_{1}, x_{2}\right)$, $U_{J}$, and $V_{J}$ are expressed in the global conordinate system $x_{1}, x_{2}$ of unit cell $Y$
} 
Some of the boundary conditions are obtained in view of the beam kinetic compatibility and equilibrium requirements at the center node of the unit cell while others are obtained in view of the requirement that the solution must be periodic with respect to the unit cell. The remaining boundary conditions are specified to insure that the solution conforms to the particular average incremental deformation applied to the unit cell.

Due to the symmetry of the unit cell with respect to the $x_{1}$ and $x_{2}$ directions, these boundary conditions simplify considerably. As a result, only the positive quadrant of the unit cell (i.e. beams 1 and 2 in Fig. 1b) needs to be considered. The corresponding boundary conditions are

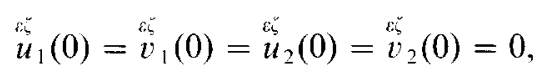

$$
\begin{aligned}
& \frac{\mathrm{d} v_{1}}{\mathrm{~d} x}(0)=\frac{\mathrm{d} v_{2}}{\mathrm{~d} x}(0) ; \quad E I_{1} \frac{\mathrm{d}^{2} v_{1}^{\varepsilon_{5}}}{\mathrm{~d} x^{2}}(0)+E I_{2} \frac{\mathrm{d}^{\varepsilon^{2} v_{2}}}{\mathrm{~d} x^{2}}(0)=0, \\
& \stackrel{\varepsilon \zeta}{u_{1}}\left(a_{1}\right)=a_{1} \delta_{i 1} \delta_{\xi 1}, \quad \stackrel{\varepsilon \zeta}{v_{1}}\left(a_{1}\right)=a_{1} \delta_{\varepsilon 2} \delta_{\xi 1} ; \quad E I_{1} \frac{\mathrm{d}^{2} v_{\xi}^{\varepsilon_{1}}}{\mathrm{~d} x^{2}}\left(a_{1}\right)=0,
\end{aligned}
$$

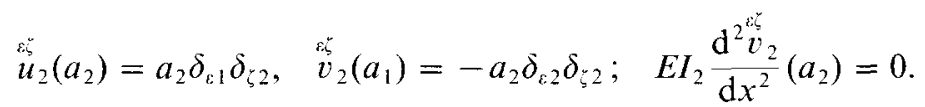

A straightforward integration of (21) subject to the boundary conditions in (22) gives the following results [recall the definition of $\beta_{\alpha}[\lambda]$ from (9)].

$$
\begin{aligned}
& \stackrel{11}{u}_{1}(x)=x, \quad v_{1}^{\prime}(x)=0, \quad u_{2}(x)=0, \quad v_{2}(x)=0, \\
& \stackrel{22}{u}_{1}(x)=0, \quad v_{1}^{22}(x)=0, \quad u_{2}^{22}(x)=x, \quad v_{2}^{22}(x)=0, \\
& \stackrel{12}{u}_{1}(x)=0, \quad{ }^{12} v_{1}(x)=\frac{N_{2}}{\Gamma}\left[-\cos \left(\beta_{1} x / a_{1}\right)+\frac{\sin \left(\beta_{1} x / a_{1}\right)}{\tan \beta_{1}}-\frac{x}{a_{1}}+1\right], \\
& \stackrel{12}{u}_{2}(x)-0, \quad v_{2}^{12}(x)=\frac{N_{1}}{\Gamma}\left[\cos \left(\beta_{2} x / a_{2}\right)-\frac{\sin \left(\beta_{2} x / a_{2}\right)}{\tan \beta_{2}}+\frac{x}{a_{2}}-1\right]-x, \\
& \stackrel{21}{u}_{1}(x)=0, \quad{ }^{21}{ }_{1}(x)=\frac{N_{2}}{\Gamma}\left[-\cos \left(\beta_{1} x / a_{1}\right)+\frac{\sin \left(\beta_{1} x / a_{1}\right)}{\tan \beta_{1}}-\frac{x}{a_{1}}+1\right]+x, \\
& \stackrel{21}{u}_{2}(x)=0, \quad{ }^{21} v_{2}(x)=\frac{N_{1}}{\Gamma}\left[\cos \left(\beta_{2} x / a_{2}\right)-\frac{\sin \left(\beta_{2} x / a_{2}\right)}{\tan \beta_{2}}+\frac{x}{a_{2}}-1\right] \\
& \Gamma \equiv \frac{N_{2}}{a_{1}}\left[1-\frac{\beta_{1}}{\tan \beta_{1}}\right]+\frac{N_{1}}{a_{2}}\left[1-\frac{\beta_{2}}{\tan \beta_{2}}\right] .
\end{aligned}
$$


From the symmetry of the unit cell with respect to the $x_{1}$ and $x_{2}$ axes,

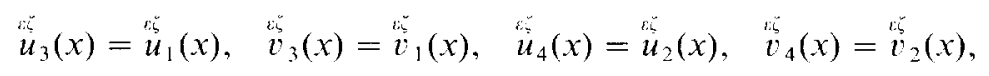

which completes the solution for the unit cell problem in (20).

Substitution of (23) and (24) into (19) yields the following expression for the homogenized incremental moduli :

$$
\begin{aligned}
& \mathscr{L}_{1111}=\frac{E A_{1}}{2 a_{2}}, \quad \mathscr{L}_{2222}=\frac{E A_{2}}{2 a_{1}}, \quad \mathscr{L}_{1122}=\mathscr{L}_{2211}=0 \\
& \mathscr{L}_{1212}=G t+\frac{1}{2 \Gamma} \frac{N_{1} N_{2}}{a_{1} a_{2}}-\frac{N_{2}}{2 a_{1}}, \quad \mathscr{L}_{2121}=G t+\frac{1}{2 \Gamma} \frac{N_{1} N_{2}}{a_{1} a_{2}}-\frac{N_{1}}{2 a_{2}}, \\
& \mathscr{L}_{1221}=G t+\frac{1}{2 \Gamma} \frac{N_{1} N_{2}}{a_{1} a_{2}}=\mathscr{L}_{2112} .
\end{aligned}
$$

For small values of $\lambda$, the homogenized material is expected to be elliptic. For adequately high values of the load parameter, the incremental moduli of the homogenized structure will lose ellipticity, which is the relevant instability that can be detected in the macroscopic (homogenized) equations of the structure.

The loss of ellipticity condition for a two-dimensional continuum occurs when for some load level $\lambda$ a unit vector $\mathbf{n}$ (with components $n_{1}, n_{2}$ ) can be found such that

$$
\operatorname{Det}\left(\mathscr{L}_{\times \beta ; \delta}[\lambda] n_{\beta} n_{\delta}\right)=0 \quad\left(n_{1}=\cos \phi, n_{2}=\sin \phi\right) .
$$

For $\lambda=0$ the acoustic tensor $\mathscr{L}_{\alpha \beta ; \delta}[0] n_{\beta} n_{\delta}$ is positive definite for any unit vector $\mathbf{n}$, as one can easily verify. Continuity with respect to $\lambda$ dictates that ellipticity is lost for the first time at $\lambda_{H}$ which satisfies

$$
\begin{gathered}
A_{11}^{H}[\lambda] n_{1}^{4}+A_{12}^{H}[\lambda] n_{1}^{2} n_{2}^{2}+A_{22}^{H}[\lambda] n_{2}^{4}>0 ; \quad 0 \leqslant \lambda<\lambda_{H} ; \\
n_{1}=\cos \phi, \quad n_{2}=\sin \phi, \quad \forall \phi \in[0,2 \pi], \\
A_{11}^{H}[\lambda] n_{1}^{4}+A_{12}^{H}[\lambda] n_{1}^{2} n_{2}^{2}+A_{22}^{H}[\lambda] n_{2}^{4}=0 ; \quad \lambda=\lambda_{H} ; \\
n_{1}=\cos \phi_{c}, \quad n_{2}=\sin \phi_{c} \\
A_{11}^{H}[\lambda] \equiv \mathscr{L}_{1111}[\lambda] \mathscr{L}_{2121}[\lambda], \quad A_{22}^{H I}[\lambda] \equiv \mathscr{L}_{1212}[\lambda] \mathscr{L}_{2222}[\lambda], \\
A_{12}^{H}[\lambda] \equiv-\left(\mathscr{L}_{1122}[\lambda]+\mathscr{L}_{1221}[\lambda]\right)\left(\mathscr{L}_{2112}[\lambda]+\mathscr{L}_{2211}[\lambda]\right) \\
+\mathscr{L}_{1111}[\lambda] \mathscr{L}_{2222}[\lambda]+\mathscr{L}_{1212}[\lambda] \mathscr{L}_{2121}[\lambda],
\end{gathered}
$$

where the expressions for $A_{\alpha \beta}^{H}[\hat{\lambda}]$ in terms of $\mathscr{L}_{\alpha \beta ; \gamma ;}$ have been derived in a straightforward fashion from (26).

Direct substitution of (25) into (27) yields, after also taking into account the definitions of $B_{\alpha}[\lambda]$ and $F_{\alpha}[\lambda]$ from (9) and the definition of $\Gamma[\lambda]$ from (23)

$$
A_{\alpha \beta}^{H}[\lambda]=\frac{1}{32 \Gamma[\lambda]} \frac{B_{1}[\lambda] B_{2}[\lambda]}{F_{1}[\lambda] F_{2}[\lambda] a_{1}^{3} a_{2}^{3}} A_{\alpha \beta}[\lambda]
$$


where $A_{\alpha \beta}[\lambda]$ are given by (12). The result in (28) implies* that $\lambda_{H}=\Lambda_{c}$, the critical load parameter corresponding to the long wavelength bifurcation mode defined in (13) and (14). It has thus been shown using a constructive proof that the loss of ellipticity instability for the homogenized (macroscopic) model corresponds to a bifurcation instability of the exact (microscopic) model when the wavelength of its buckling mode is much larger than the unit cell $\left(L_{\alpha} \gg a_{\alpha}\right.$ or equivalently $\left.\omega_{\alpha} a_{\alpha} \ll 1\right)$.

The above result, i.e. that the long wavelength eigenmode buckling load for a nonlinear periodic medium coincides with the load at which the homogenized moduli of the material lose their ellipticity is a general theorem holding for incrementally linear period media as shown in a very general three-dimensional context by GEYMONAT $e t$ al. $(1990,1992)$. An earlier constructive proof of this result in the case of layered composites was given by TRIANTAFYLLIDIS and MAKER (1985).

\section{Results And Discussion}

The logical starting point for the results is the parametrization of the model. Instead of the six geometric parameters $A_{x}, I_{\alpha}$ and $a_{x}$ (areas, moments of inertia and lengths of beams in the $x_{1}$ and $x_{2}$ directions), the following parameters are introduced

$$
\begin{array}{llll}
c \equiv\left(\left(c_{1}\right)^{2}+\left(c_{2}\right)^{2}\right)^{1 / 2}, & \chi_{c} \equiv \tan ^{-1}\left[c_{2} / c_{1}\right] ; & c_{x} \equiv 12 I_{\alpha} /\left(A_{\alpha}\right)^{2}, \\
r \equiv\left(\left(r_{1}\right)^{2}+\left(r_{2}\right)^{2}\right)^{1 / 2}, & \chi_{r} \equiv \tan ^{-1}\left[r_{2} / r_{1}\right] ; & r_{x} \equiv\left(12 I_{x} / A_{\alpha}\right)^{1 / 2} / a_{\alpha}, \\
a \equiv\left(\left(a_{1}\right)^{2}+\left(a_{2}\right)^{2}\right)^{1 / 2}, & \chi_{a} \equiv \tan ^{-1}\left[a_{2} / a_{1}\right] . &
\end{array}
$$

The $c_{1}, c_{2}$ and $c$ parameters in (29) are termed shape parameters. In the case of a beam with a rectangular cross-section, $c_{\alpha}$ simplifies to $h_{\alpha} / b_{\alpha}$ where $h_{\alpha}$ is the beam's inplane thickness and $b_{\alpha}$ is the out-of-plane thickness. The shape parameters tend to increase with more efficient beam cross-section designs, such as I-beams, that place most of the cross-sectional area as far from the neutral axis as possible. The $r_{1}, r_{2}$ and $r$ parameters in (29) are termed slenderness parameters. For a beam with a rectangular cross-section, $r_{\alpha}$ simplifies to $h_{\alpha} / a_{x}$. The slenderness parameters should be of the order of $10^{-1}$ or smaller because the model derivation involves slender beam assumptions. Finally, $2 a$ is the diagonal size of the unit cell.

At this point the dimensionless interstitial stiffness parameter $g$ is introduced

$$
g \equiv \frac{G t}{E a} .
$$

The dimensionless load parameter $\lambda$ to be used in the model is defined as follows

$$
N_{1} \equiv 2 E a_{2} a \lambda \cos \theta, \quad N_{2} \equiv 2 E a_{1} a \lambda \sin \theta,
$$

where $\theta$ is termed the load ratio parameter. With the above definition, the dimensionless compressive stresses $\sigma_{1} / E$ and $\sigma_{2} / E$ applied on the model in the $x_{1}$ and $x_{2}$ directions respectively are

\footnotetext{
${ }^{*} B_{x}[\lambda], F_{x}[\lambda]$ and $\Gamma_{x}[\lambda]$ have no roots in the range of interest $0 \leqslant \lambda \leqslant \lambda_{i t}$ for all cases considered.
} 


$$
\frac{\sigma_{1}}{E}=\frac{N_{1} /\left(2 a_{2}\right)}{E a}=\lambda \cos \theta, \quad \frac{\sigma_{2}}{E}=\frac{N_{2} /\left(2 a_{1}\right)}{E a}=\lambda \sin \theta
$$

where $\sigma_{1}=N_{1} /\left(2 a_{2} a\right)$ and $\sigma_{2}=N_{2} /\left(2 a_{1} a\right)$ are the dimensionless average principal stresses applied along the $x_{1}$ and $x_{2}$ directions, respectively.

The results of the critical load dependence on various microstructure geometries will be shown in the form of curves in $\sigma_{1} / E-\sigma_{2} / E$ diagrams. Because of the choice of parametrization made in (31) and (32), these plots are polar plots of the critical load parameter $\lambda_{\mathrm{c}}$ versus $\theta$. In all the calculations reported here $E=1$ and $a=\sqrt{2}$, thus fixing the units of force and length for the subsequent results.

At first, attention is focussed on the stability of the grillage in the absence of interstitial material $(g=0)$. The critical load $\lambda_{c}$ is found from $(10)$ as the minimum height of the surface $\hat{\lambda}\left(\sqrt{s_{1}}, \sqrt{s_{2}}\right)$ with the dimensionless mode wavenumber parameters, $s_{x}$ defined in $(9), s_{\alpha} \equiv\left(\sin \left[\omega_{x} a_{x}\right]\right)^{2}$. For slender beams, a typical graph of $\hat{\lambda}$ as a function of $\sqrt{s_{1}}$ and $\sqrt{s_{2}}$ is given in Fig. 2a $\left(\chi_{u t}=\pi / 4, c=\sqrt{2}, \chi_{c}=\pi / 4, r=0.2\right.$, $\left.\chi_{r}=\pi / 4\right)$ for $\theta=\pi / 4$. The wanted minimum $\lambda_{\mathrm{c}}$ of $\hat{\lambda}\left(\sqrt{s_{1}}, \sqrt{s_{2}}\right)$ occurs at the origin $\sqrt{s_{1}}=\sqrt{s_{2}}=0$, at the vicinity of which the surface $\lambda$ is singular and forms a hump. Since the minimum of $\hat{\lambda}$ occurs at the origin, $\lambda_{c}=\Lambda_{c}$, where $\Lambda_{c}$ is defined in (14). For slender beams one always finds that $\Lambda_{\mathrm{c}}=\Lambda_{1}$ or $\Lambda_{2}$, thus implying $\mathbf{n}_{\mathrm{c}}=\mathbf{i}$ or $\mathbf{j}$, i.e. that the macroscopic characteristic directions for the failure of the structure coincide with the principal stress axes. For much stubbier beams a typical graph of $\hat{\lambda}$ as a function of $\sqrt{s_{1}}$ and $\sqrt{s_{2}}$ is given in Fig. $2 \mathrm{~b}\left(\chi_{a}=\pi / 4, c=\sqrt{2}, \chi_{c}=\pi / 4, r=5.0, \chi_{r}=\pi / 4\right)$ for

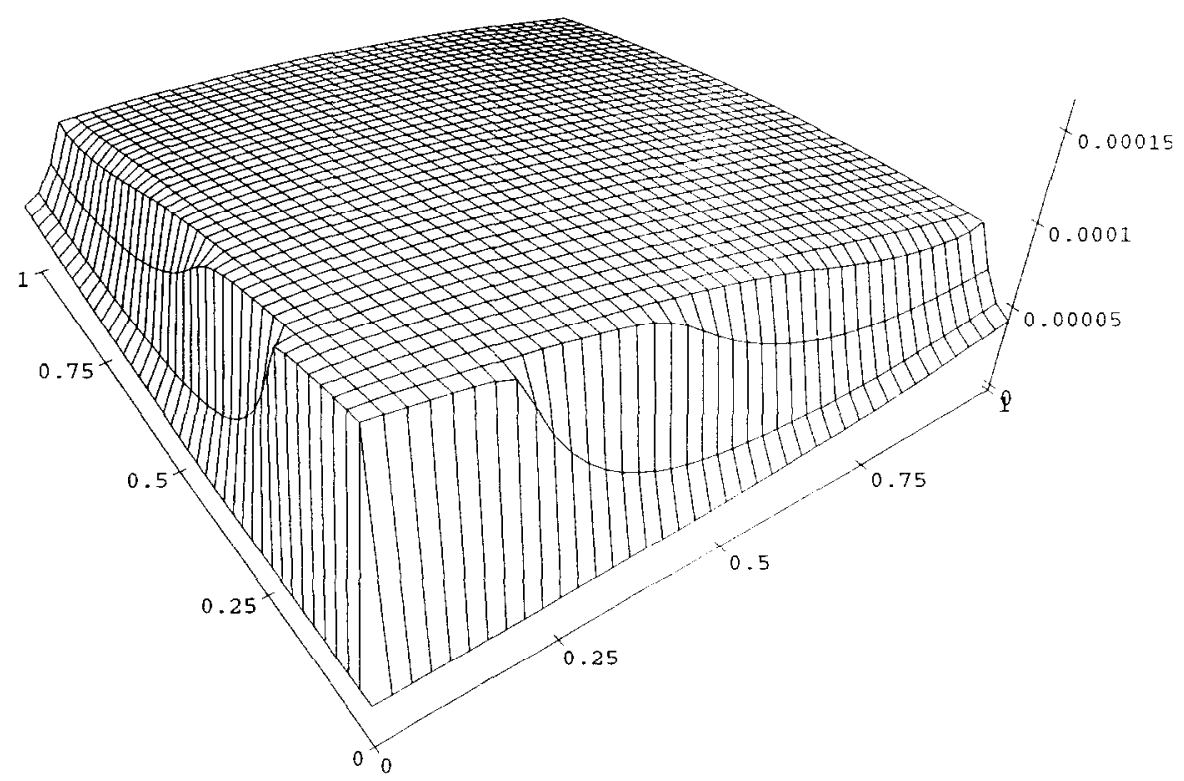

FIG. 2a. Typical buckling load $\hat{\lambda}$ versus mode wavenumber parameters $\sqrt{s_{1}}, \sqrt{s_{2}}$ surface for a small beam slenderness parameter $r$ when the interstitial stiffness is ignored $(g=0)$. Notice that the minimum of $\hat{\lambda}$ occurs at the origin $(0,0)$, indicating that the critical mode is a long wavelength mode. 


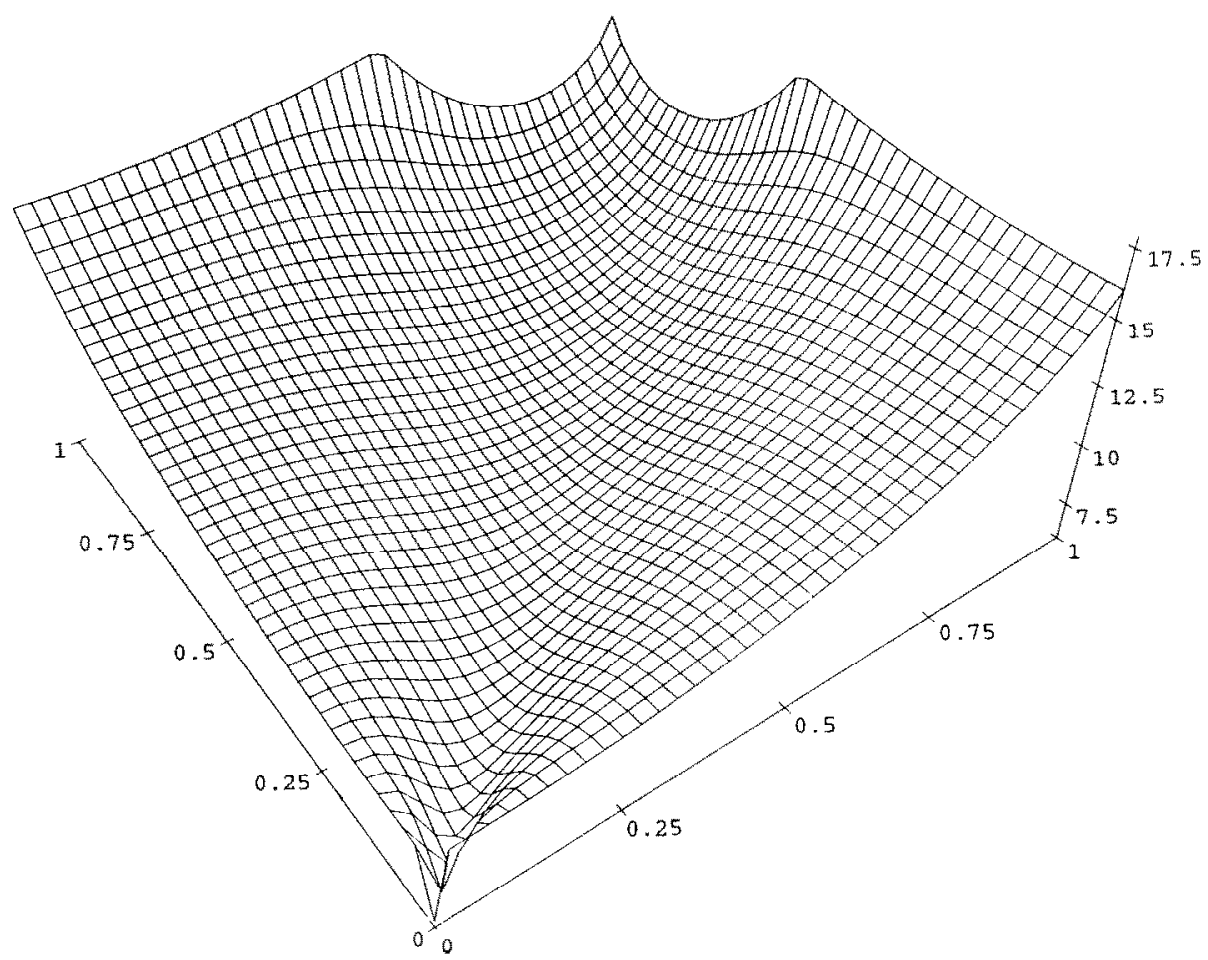

Fig. 2b. Typical buckling load $\hat{h}$ versus node wavenumber parameters $\sqrt{s_{1}}, \sqrt{s_{2}}$ surface for a large bean slenderness parameter $r$ when the interstitial stiffness is ignored $(g=0)$. Notice that the minimum of $\hat{\lambda}$ occurs at the origin $(0,0)$, indicating that the critical mode is a long wavelength mode.

$\theta=\pi / 4$. Once again, the wanted critical load $\lambda_{c}$ occurs at the origin $\sqrt{s_{1}}=\sqrt{s_{2}}=0$, at the vicinity of which the surface $\hat{\lambda}$ is singular and forms a valley. Since the minimum of $\hat{\lambda}$ occurs at the origin, $\lambda_{\mathrm{c}}=\Lambda_{\mathrm{c}}$. Note that in contrast to the slender beam case, $\Lambda_{c}=\Lambda_{\infty}$, which implies a macroscopic characteristic direction for the failure of the structure that is not in one of the principal stress directions. This can be easily seen from Fig. $2 b$, where if one approaches the origin from along the bottom of the valley (in this case with $s_{1}=s_{2}$ ), one obtains the value $\lambda_{\mathrm{c}}=\Lambda_{\mathrm{c}}$. It should be mentioned at this point that $\hat{\lambda}(0,0)$ (which corresponds to the periodic buckling mode of the structure, i.e. the mode where the wavelengths in both directions are integral multiples of the respective unit cell dimensions) is always considerably higher than any value of $\hat{\Lambda}$ and hence is omitted in Figs $2 a$ and $2 b$ as well as in Figs $8 a$ and $8 b$.

The common feature for all the critical load calculations in grillages with no interstitial stiffness $(g=0)$ is that the critical buckling mode always corresponds to the long wavelength mode $\left(L_{\alpha} / a_{x}=2 \pi /\left(\omega_{x} a_{\alpha}\right) \rightarrow \infty\right)$. This result is to be expected, since virtually all of the strain energy of the buckled configuration is stored through beam bending. Because the long wavelength modes correspond to vanishingly small beam curvatures, they thus minimize the energy stored per unit cell of the structure. 
The same result was found by BendseE and Triantafyllidis (1990), who used a grillage model in their study of optimal microstructures.

For the grillages with no interstitial material $(g=0)$ typical dimensionless critical stress $\left(\sigma_{1} / E\right.$ and $\left.\sigma_{2} / E\right)$ diagrams are given in Figs $3 \mathrm{a}$ and $3 \mathrm{~b}$ for thin and stubby beams, respectively. More specifically, Fig. 3a corresponds to the thin beam grillage used for Fig. $2 \mathrm{a}\left(\chi_{a}=\pi / 4, c=\sqrt{2}, \chi_{c}=\pi / 4, r=0.2, \chi_{r}=\pi / 4\right)$ for $0 \leqslant \theta \leqslant \pi / 2$. The chain-dotted (--) line in Fig. 3a corresponds to $\Lambda_{1}(\theta)$ and the chain-dashed (- - ) line corresponds to $\Lambda_{2}(\theta)$. The shaded area in Fig. 3a contains all the stress states for which the grillage is stable. The chain-dotted and chain-dashed parts of the boundary of the shaded area contain all of the stress states corresponding to the onset of the first bifurcation. The first bifurcation coincides with the first loss of ellipticity for the homogenized moduli, since $\lambda_{\mathrm{c}}=\Lambda_{\mathrm{c}}$. The critical stresses in Fig. $3 \mathrm{~b}$ correspond to the stubby beam grillage used for Fig. $2 \mathrm{~b}\left(\chi_{a}=\pi / 4, c=\sqrt{2}, \chi_{c}=\pi / 4, r=5.0\right.$, $\chi_{r}=\pi / 4$ ) for $0 \leqslant 0 \leqslant \pi / 2$. Similarly to the previous figure the chain-dotted and chaindashed lines correspond to $\Lambda_{1}(\theta)$ and $\Lambda_{2}(\theta)$, respectively, while the dashed ( - ..) line corresponds to $\Lambda_{1}(\theta)$ which as expected for the stubby beams satisfies $\Lambda_{,}(\theta)<\Lambda_{1}(\theta), \Lambda_{2}(\theta)$. The shaded area containing the origin and bounded by $\Lambda_{,}(\theta)$

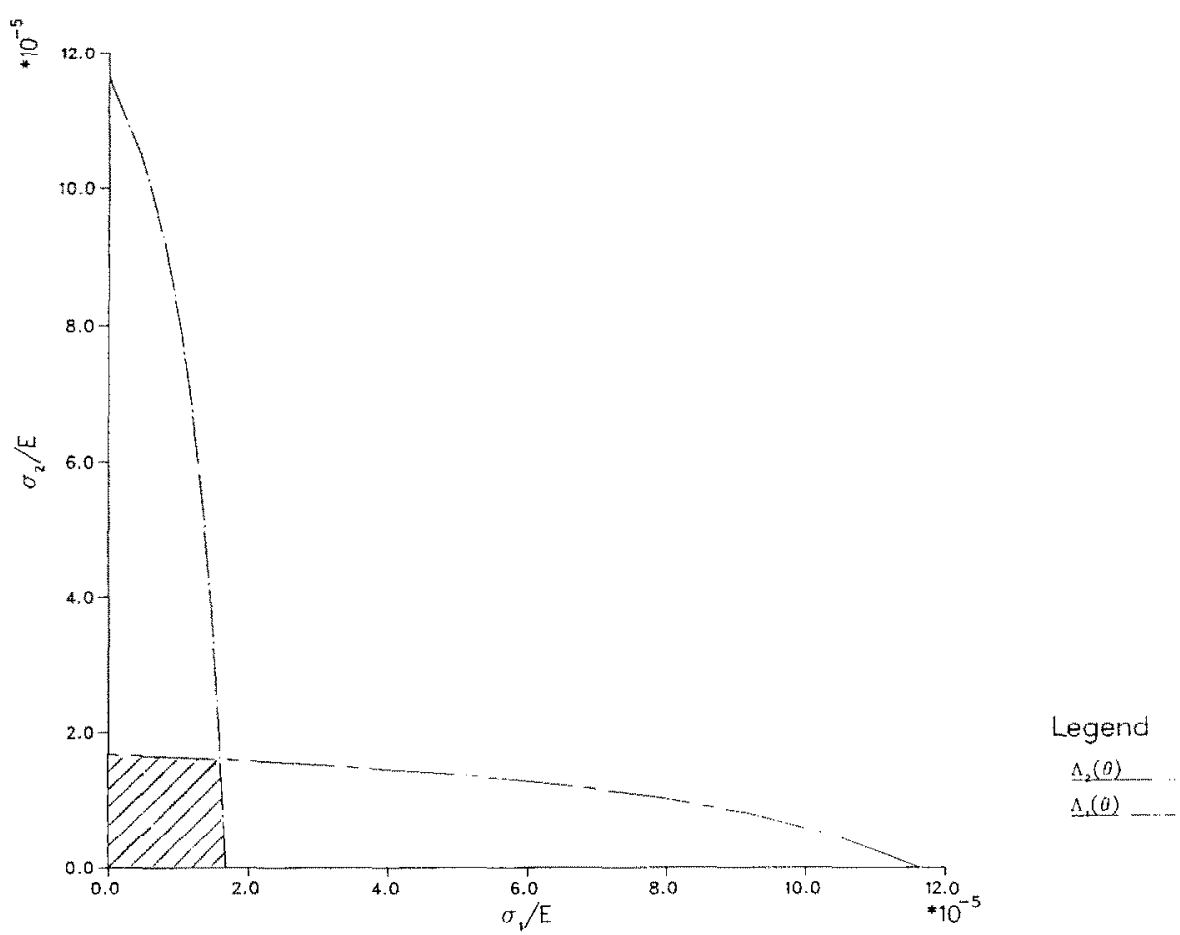

FIG. 3a. Typical critical dimensionless stress diagram for grillages with slender beams when interstitial stiffness is ignored $(g=0)$. The $(-\cdot)$ line corresponds to the long wavelength mode $\Lambda_{1}(\theta)$, and the $\left(\right.$ line corresponds to the long wavelength mode $\Lambda_{2}(0)$. The shaded area contains all the strain states for which the grillage is stable. 


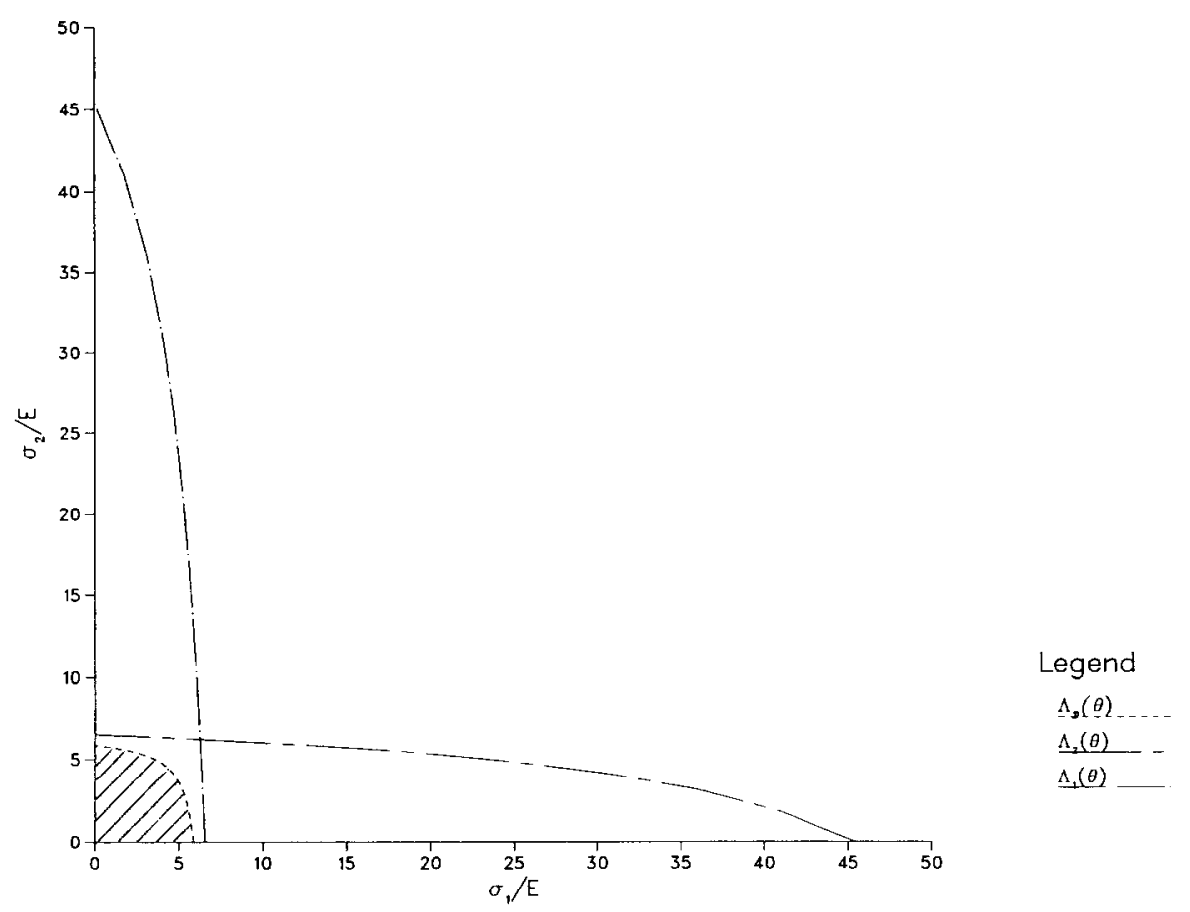

FIG. 3b. Typical critical dimensionless stress diagram for grillages with stubby beams when interstitial stiffness is ignored $(g=0)$. The $(-\cdot-)$ line corresponds to the long wavelength mode $\Lambda_{1}(\theta)$, the $(-\cdots)$ line corresponds to the long wavelength mode $\Lambda_{2}(\theta)$ and the (---) line corresponds to the long wavelength mode $\Lambda_{\mathscr{Z}}(0)$. The shaded area contains all the strain states for which the grillage is stable.

contains all the stable stress states for the grillage and coincides with the domain of ellipticity for the homogenized moduli since $\lambda_{\mathrm{c}}=\Lambda_{\mathrm{c}}$.

The effect of the various geometric parameters of the grillage on the stability region of the structure in the $\sigma_{1} / E-\sigma_{2} / E$ space is investigated in Figs 4-7b.

The influence of the shape of the grillage on its stability region is studied in Fig. 4 in the case of a thin beam grillage $\left(25^{\circ} \leqslant \chi_{a} \leqslant 45^{\circ}, c=\sqrt{2}, \chi_{c}=\pi / 4, r=0.2, \chi_{r}=\pi / 4\right)$ for $0 \leqslant \theta \leqslant \pi / 2$. Notice that the structure made up of square unit cells $\left(a_{1}=a_{2}\right.$ or $\left.\chi_{a}=\pi / 4\right)$ possesses the largest region of stable stress states. The stable regions corresponding to the other values of $\chi_{a}$ are nested one inside the other as the unit cell shape becomes more and more elongated. This behavior is at first surprising, because although the effect of moving $\chi_{a}$ away from $\pi / 4$ consists of weakening beams in one direction, it actually strengthens those in the other direction. However, the $x$ - and $y$ responses of the structure are coupled due to the fact that beams in the $x$-direction and $y$-direction are fastened together by welded joints. This means that when the bifurcation mode involves rotations of the joints (which is the case for the long wavelength mode) the strengths of the beams in both directions affect the critical load. This is the reason the unit cell shows weakening for all load ratio parameters, including the range of $\theta$ where the load is more concentrated in the apparently strengthened direction. Qualitatively similar results can be obtained for stubbier beams. 


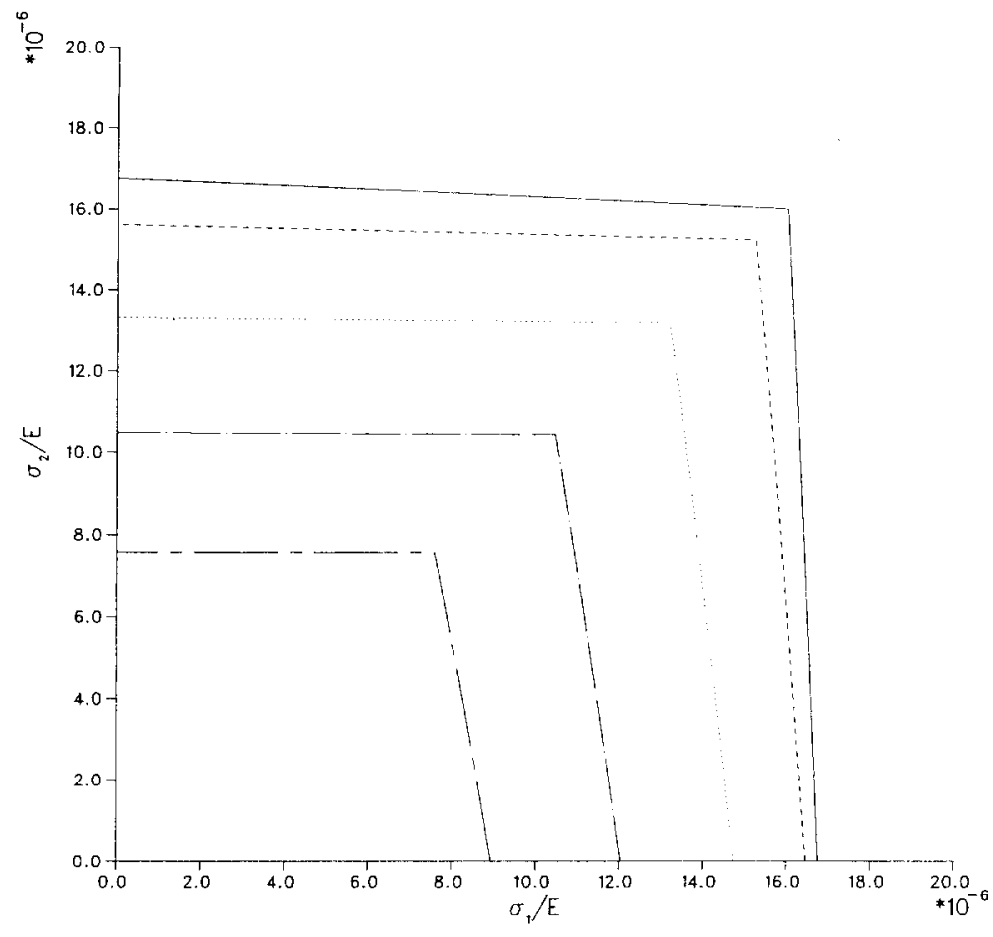

Legend

$x_{a}=45^{\circ}$

$x=40^{\circ}$

$x_{0}=35^{\circ}$

$x_{0}=30^{\circ}$

$x_{0}=25^{\circ}$

$\tan \left(x_{0}\right)=a_{2} / a_{1}$

FIG. 4. Dependence of the stability region of a slender beam grillage with no interstitial stiffness $(g=0)$ on the shape of the unit cell as measured by the angle $\chi_{x}$ formed between the cell diagonal and the $x_{1}$ axis.

The influence of the stubbiness of the beams, as described by the $r$ and $\chi_{r}$ parameters, is investigated in Figs 5a and 5b. More specifically, Fig. 5a explores the dependence on $\chi_{r}$ of the stability region in the $\sigma_{1} / E-\sigma_{2} / E$ space for a slender beam grillage $\left(\chi_{a}=\pi / 4, c=\sqrt{2}, \chi_{c}=\pi / 4, r=0.2,25 \leqslant \chi_{r} \leqslant 45^{\circ}\right)$ for $0 \leqslant \theta \leqslant \pi / 2$. Similarly to the result in Fig. 4, and for the same reason, the stability boundaries are nested one inside the other, with the largest region of stable stress states corresponding to the model with $\chi_{r}=\pi / 4$. In view of the slenderness of the beams, the stable regions are of the type presented in Fig. 3a, since their stability boundaries are formed by the $\Lambda_{1}(\theta)$ and $\Lambda_{2}(\theta)$ curves. The effect of $\chi_{r}$ on the stability of grillages with stubbier beams is illustrated in Fig. $5 \mathrm{~b}\left(\chi_{a}=\pi / 4, c=\sqrt{2}, \chi_{c}=\pi / 4, r=5.0,25^{\circ} \leqslant \chi_{r} \leqslant 45^{\circ}\right)$ for $0 \leqslant \theta \leqslant \pi / 2$ where onc can see the transition from a stability region boundary determined by $\Lambda_{g}(\theta)$ (for $\chi_{r}=\pi / 4$ ) to a stability region whose boundary is determined by $\Lambda_{1}(\theta)$ and $\Lambda_{2}(\theta)$ (for $\chi_{r}=25^{\circ}$ ). Again, the stability regions are sequentially nested as expected inside the $\chi_{r}=\pi / 4$ curve. The effect of changing $r$ while keeping $\chi_{r}$ constant is easily predictable and produces stability regions nested inside one another as $r$ decreases.

The effect of the beam shape constants $c$ and $\chi_{c}$ on the stability of the grillage is investigated in Fig. 6. For slender beams $\left(\chi_{a}=\pi / 4, c=\sqrt{2}, 25 \leqslant \chi_{c} \leqslant 45^{\circ}, r=0.2\right.$, $\chi_{r}=\pi / 4$ ) the effects of the parameters $\chi_{r}$ are shown in Fig. 6 for $0 \leqslant \theta \leqslant \pi / 2$. Unlike 
the effects of $\chi_{a}$ or $\chi_{r}$, the stable regions are not sequentially nested as $\chi_{c}$ moves away from $\pi / 4$. However, the effects of $\chi_{c}$ are negligible compared to those of $\chi_{a}$ or $\chi_{r}$. The effect of changing $c$ predictably produces stability regions nested inside one another as $c$ increases. This happens because holding $r$ constant and increasing $c$ reduces the beam cross-sectional areas, thus weaking the structure.

From the results shown in Figs $4,5 \mathrm{a}, 5 \mathrm{~b}$ and 6 , one concludes that the beam stubbiness parameters $r$ and $\chi_{r}$ determine the type of failure mode for the structure [i.e. whether $\lambda_{\mathrm{c}}(\theta)=\Lambda_{\mathrm{r}}(\theta)$, or $\lambda_{\mathrm{c}}(\theta)=\Lambda_{1}(\theta)$ or $\Lambda_{2}(\theta)$ ] and hence the shape of the stable region in the $\sigma_{1} / E-\sigma_{2} / E$ space. Upon examining a very wide range of the grillage parameters for the case of $g=0$, it has been found that $\lambda_{\mathrm{c}}=\Lambda_{\mathscr{c}}(\theta)$ solutions can only occur when $r$ becomes sufficiently large. This transition between failure modes can be seen as the effect of the beam stubbiness $r$ on the characteristic direction at the loss of ellipticity, $\phi_{\mathrm{c}} \equiv \tan ^{-1}\left(n_{2 \mathrm{c}} / n_{\mathrm{lc}}\right)$, depicted in Fig. 7a for a structure with $\chi_{a}=\pi / 4$, $c=\sqrt{2}, \chi_{i}=\pi / 4,1.0 \leqslant r \leqslant 8.0$, and $\chi_{r}=\pi / 4$, for $\theta=40^{\circ}$. As expected, there is a more or less abrupt transition from the $\lambda_{c}=\Lambda_{1}$ or $\Lambda_{2}$ slender beam type mode (with $\phi_{c}=0$ or $\phi_{c}=\pi / 2$ ) to the $\lambda_{c}=\Lambda_{c}$ stubby beam type mode (with $0<\phi_{c}<\pi / 2$ ). The load ratio parameter was intentionally chosen as $\theta=40^{\circ}$ because the symmetry of the above model about the $x_{1}=x_{2}$ diagonal prevents the model from achieving any

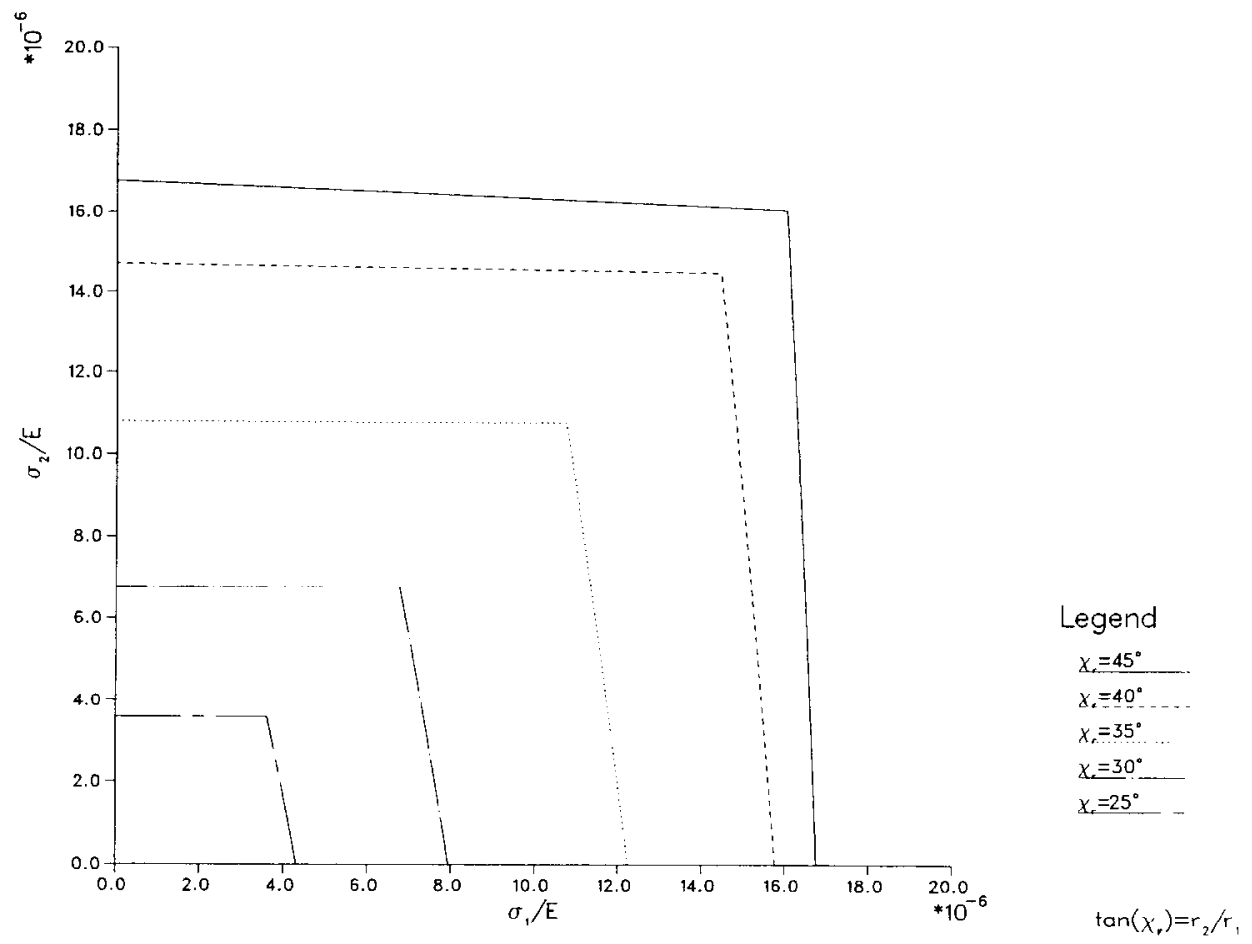

FIG. 5a. Dependence of the stability region of a slender beam grillage with no interstitial stiffness $(g=0)$ on the differential strengthening of beams in each direction as measured by the slenderness angle $\chi_{r}\left(\chi_{r}<\pi / 4\right.$ corresponds to stronger beams in the $x_{1}$ direction). 


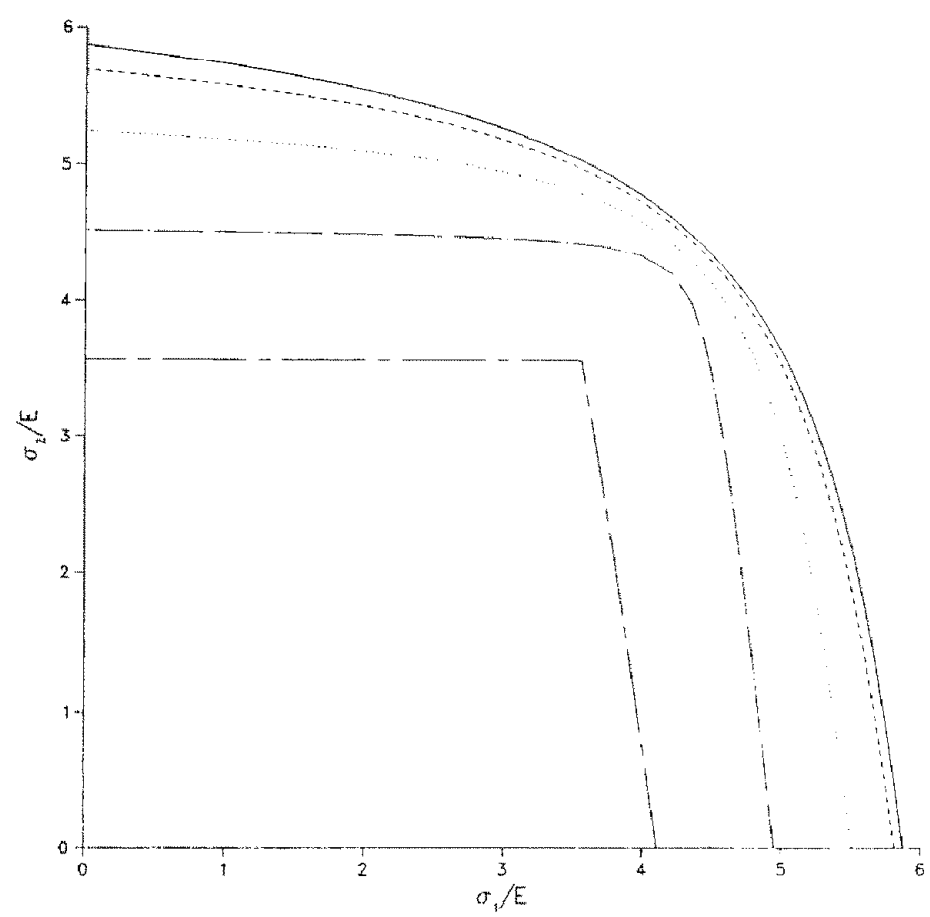

Legend

$x=45^{\circ}$

$x=39^{\circ}$

$x=36^{\circ}$

$x_{1}=33^{\circ}$

FiG. Sb. Dependence of the stability region of a stubby beam grillage with no interstitial stifthess $(a=0)$ on the differential strengthening of beams in cach direction as measured by the slenderness angle $\chi_{r}\left(\chi_{,}<\pi / 4\right.$ corresponds to stubbier beams in the $x_{1}$ dircetion).

$\phi_{0}$ other than $\pi / 4$ (or 0 ) if $\theta=\pi / 4$. For stubby beams, there is a strong dependence of $\phi_{c}$ on the load ratio parameter $\theta$ as typified by Fig. $7 \mathrm{~b}\left(\chi_{a}=\pi / 4, c=\sqrt{2}, \chi_{r}=\pi / 4\right.$, $r=4.25, \chi_{r}-\pi / 4$ ) for $0 \leqslant 0 \leqslant \pi / 2$.

In all the above results, where the interstitial stiffness is neglected $(g=0)$, the stability region in the $\sigma_{1} / E-\sigma_{2} / E$ space (i.e. the stress states for which buckling is excluded) coincides with the ellipticity domain for the homogenized equations, since the critical mode is always the long wavelength mode. The situation is quite different for $g \neq 0$ where for adequately large values of $g$ a local buckling mode can precede the global one, i.e. at $\lambda_{c},\left(\sqrt{ } s_{1}, \sqrt{s_{2}}\right)_{\mathrm{c}} \neq(0,0)$. Some typical graphs of $\hat{i}\left(\sqrt{s_{1}}, \sqrt{ } s_{2}\right)$, for the case of $g \neq 0$ are shown in Figs $8 \mathrm{a}$ and $8 \mathrm{~b}$. The $\lambda\left(\sqrt{s_{1}}, \sqrt{s_{2}}\right)$, surface in Fig. 8 a corresponds to a grillage with the same beams as in Fig. $2 a\left(\chi_{i t}=\pi / 4, c=\sqrt{2}\right.$, $\chi_{r}=\pi / 4, r=0.2, \chi_{r}=\pi / 4$ ) for $\theta=\pi / 4$, but with a rather large interstitial stiffness parameter $g=1.0$. The critical load in this case is achieved simultaneously for $\left(\sqrt{s_{1}}, \sqrt{s_{2}}\right)_{c}=(0,1),\left(\sqrt{s_{1}}, \sqrt{s_{2}}\right)_{c}=(1,0)$, and $\left(\sqrt{s_{1}}, \sqrt{s_{2}}\right)_{c}=(1,1)$, i.e. $\lambda_{c}=$ $\hat{\lambda}(0,1)=\hat{\lambda}(1,0)=\hat{\lambda}(1,1)$ (an unusual type of situation due to the symmetry of the geometry of the unit cell and the symmetry of the loading, about the $x_{1}=x_{2}$ diagonal). The above results correspond to antisymmetric type modes since $\sin ^{2}\left(\omega_{x} a_{x}\right)=1$ (for $x=1$ or 2$)$ for which the longest corresponding wavelengths are $L_{x} / a_{x}=2 \pi /\left(\omega_{x} a_{x}\right)=$ $2 \pi /(\pi / 2)=4$. 

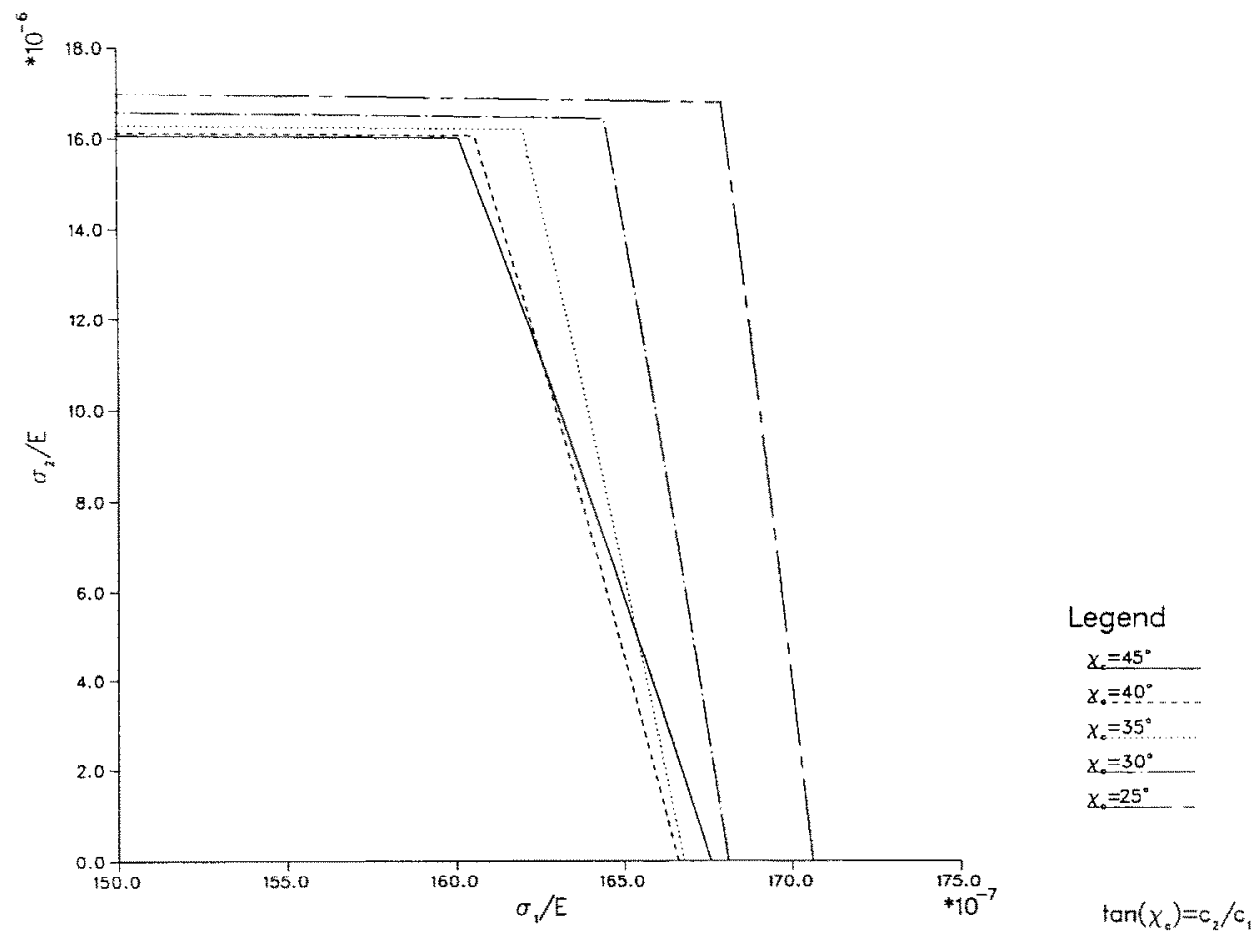

FIG. 6. Dependence of the stability region of a slender beam grillage with no interstitial stiffness $(g=0)$ on the differential strengthening of beams in each direction as measured by the beam shape angle $\chi_{i}\left(\chi_{i}<\pi / 4\right.$ corresponds to cross-sections with a more efficient cross-section design in the $x_{1}$ direction, i.e. $c_{1}>c_{2}$ ).

Calculations of $\hat{\lambda}\left(\sqrt{s_{1}}, \sqrt{s_{2}}\right)$ for $\left(\sqrt{s_{1}}, \sqrt{s_{2}}\right)$ over $[0,1] \times[0,1]$ were performed here for a great variety of unit cell geometries and interstitial properties. For almost all of the models examined, the critical mode was found in one of the four corners of the $\left(\sqrt{s_{1}}, \sqrt{s_{2}}\right)$ domain of definition of $\hat{\lambda}$, i.e. $\sqrt{s_{1}}=0$ or 1 and $\sqrt{s_{2}}=0$ or 1 . Some models, however, possess critical modes not corresponding to one of the four corners of $[0,1] \times[0,1]$, but rather to a point interior to this region. Figure $8 b$ depicts the $\hat{\lambda}\left(\sqrt{s_{1}}\right.$, $\left.\sqrt{s_{2}}\right)$ surface for a grillage with $\chi_{a}=\pi / 4, c=1.35, \chi_{i}=\pi / 4, r=0.1225, \chi_{r}=$ 11.5, and $g=1.0$, for $\theta=0$, showing the minimum load at the interior point $\left(\sqrt{s_{1}}, \sqrt{s_{2}}\right)_{c} \cong(0.84,0.15)$. Models which display this type of behavior belong to the limited class of unit cells whose beam properties are significantly stronger in one direction than the other. Furthermore, these models must be loaded solely in the strong direction in order to obtain the critical mode at an interior point, i.e. the interior point mode is very sensitive to the load ratio parameter $\theta$. For example, for the model used to generate Fig. $8 \mathrm{~b}$, as soon as $\theta$ deviates from 0 , the critical mode moves to the $\left(\sqrt{s_{1}}, \sqrt{s_{2}}\right)=(1,0)$ corner.

For the more common grillages where the minimum of $\hat{\lambda}\left(\sqrt{s_{1}}, \sqrt{s_{2}} ; \theta\right)$ occurs at one of the four corners of the $[0,1] \times[0,1]$ domain, the set of all stable stress states can be found as the intersection of the domain for which a long wavelength bifurcation 


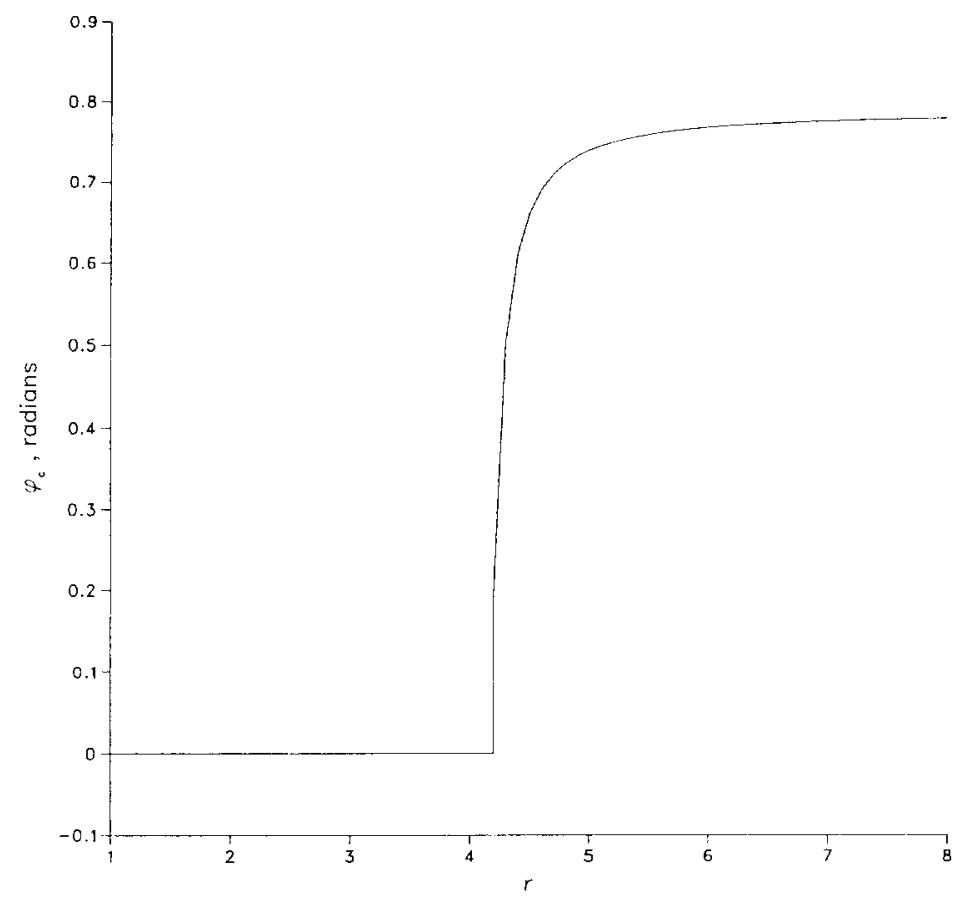

$\tan (f)=n_{2} / r$.

FIG. 7a. Effect of the beam slenderness parameter $r$ on the loss of ellipticity direction $\phi$, for grillages with no interstitial stiffness $(g=0)$ at fixed $\theta=40$.

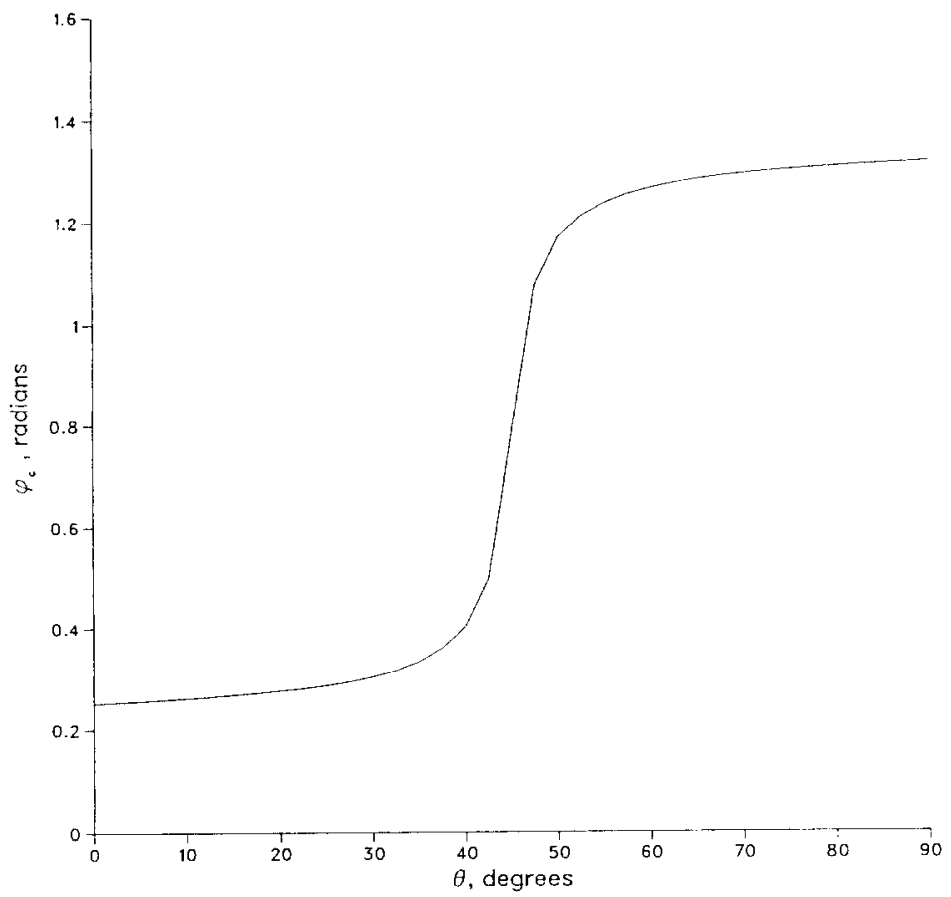

$$
\tan (\varphi)=n_{7} / n
$$

FIG. 7b. Effect of the load ratio parameter $\theta$ on the loss of ellipticity direction $\phi$, for a grillage with no interstitial stiffness $(g=0)$ and $r=4.25$. 


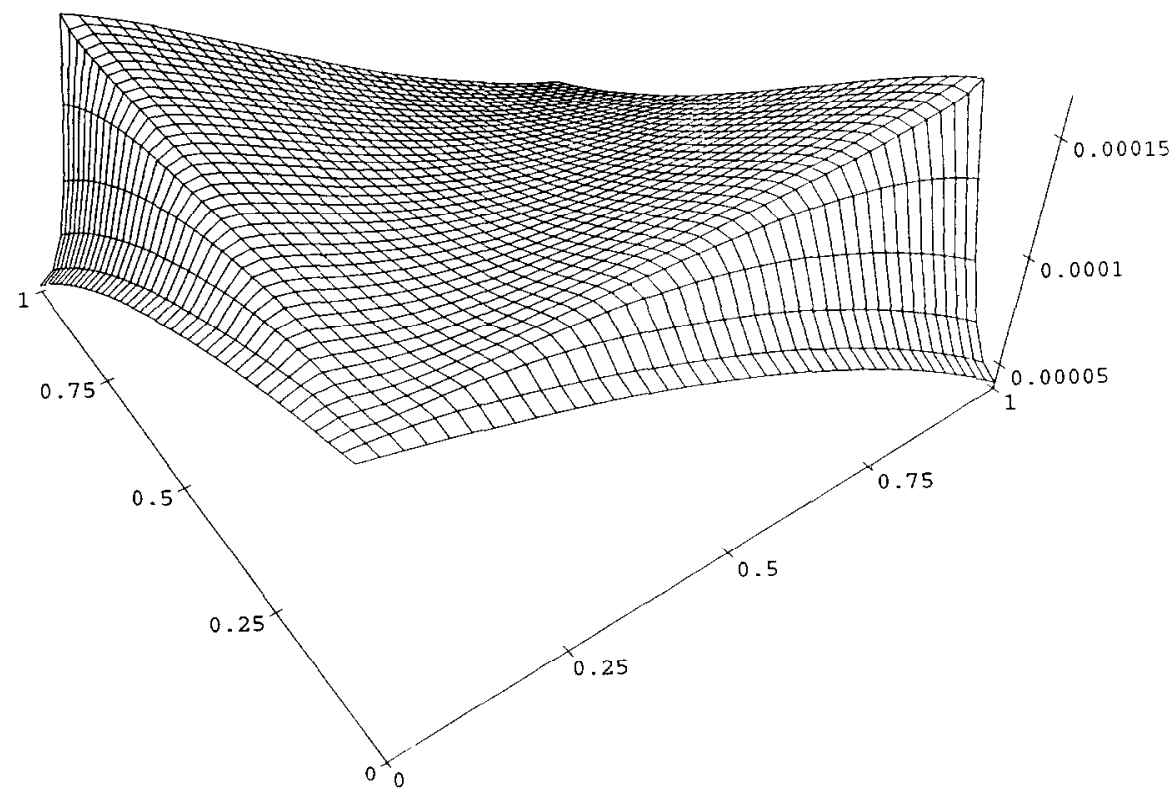

Fig. 8a. Typical buckling load $\hat{\lambda}$ versus mode wavenumber parameters $\sqrt{s_{1}}, \sqrt{s_{2}}$ surface for a grillage with interstitial stiffness $g \neq 0$. Notice that the minimum $\hat{\lambda}$ is no longer at the origin but at one of the other cormers of the $[0,1] \times[0,1]$ domain of definition of the wavenumber parameters, indicating that the critical mode is a local one.

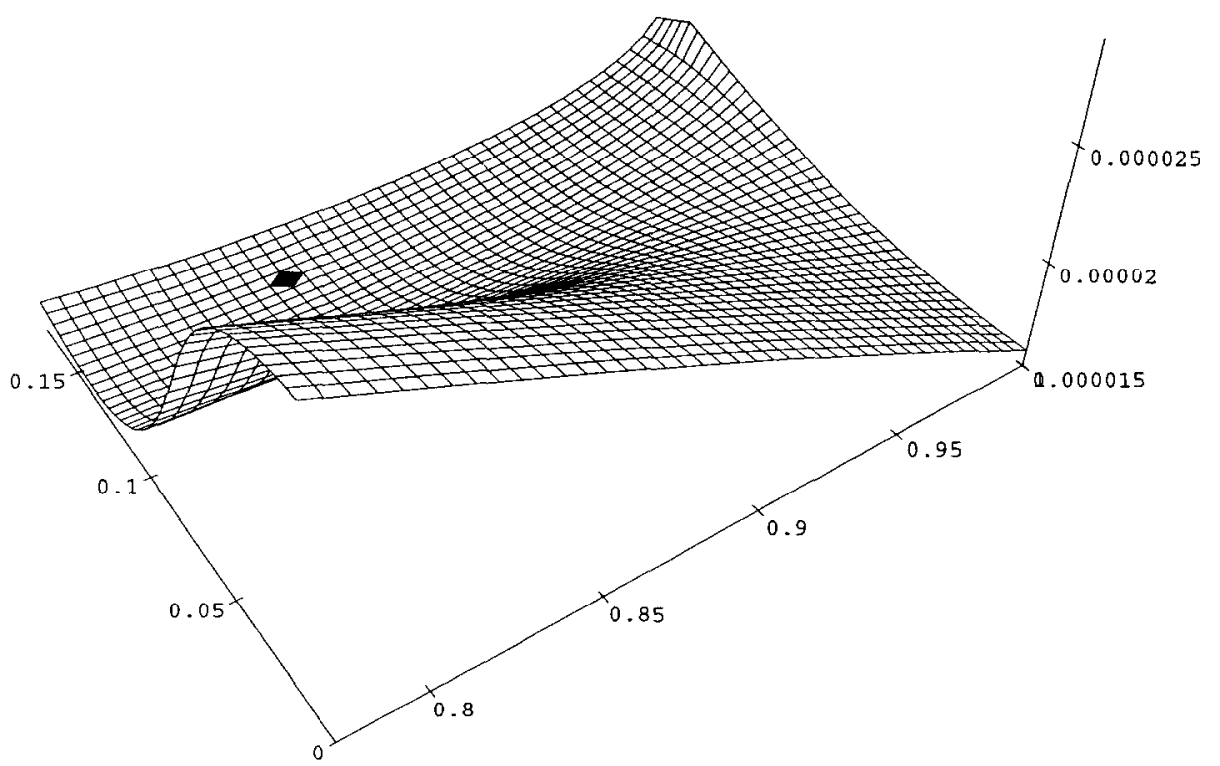

FIG. 8b. Under exceptional circumstances, for grillage models with interstitial stiffness $g \neq 0$, one can find the minimum in the critical load surface $\hat{\lambda}\left(\sqrt{s_{1}}, \sqrt{s_{2}}\right)$ to be an interior point (dark spot) away from the boundary of the $[0,1] \times[0,1]$ domain of the wavenumber parameters. This indicates that the critical mode is a local one whose wavelength has no special relation to the unit cell dimensions. 


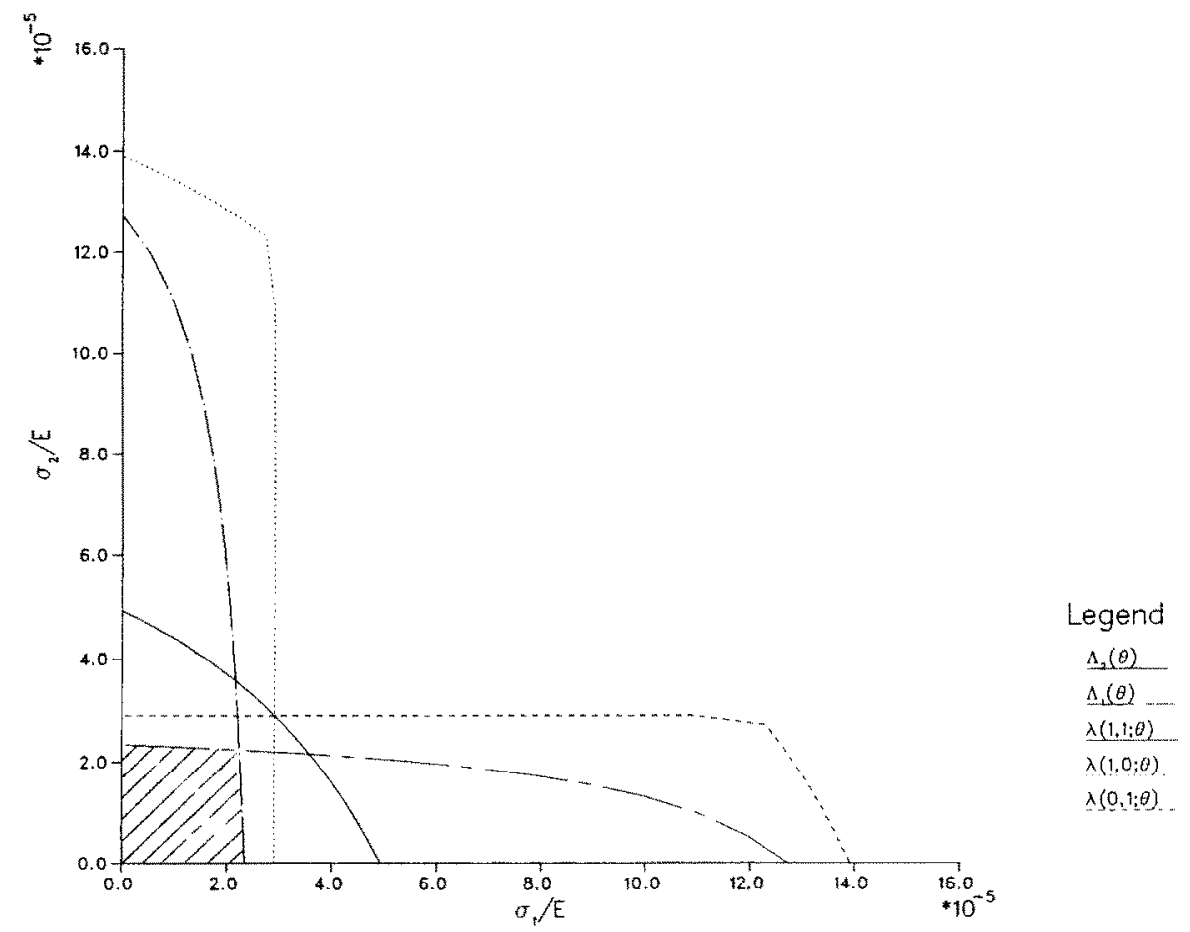

FIG. 9a. Typical critical dimensionless stress diagram for slender beam grillages with low interstitial stifness $g=1.0 \times 10^{-5}$. The $(-\cdots)$ and $(-\infty)$ lines correspond to the long wavelength mode curves $\Lambda_{1}(0)$ and $\Lambda_{2}(0)$, while the $(-\cdots),(\cdots)$ and $(-)$ lines correspond to the finite mode curves $\hat{\lambda}(0,1 ; 0), \hat{\gamma}(1,0 ; 0)$ and $f(1,1 ; 0)$, respectively. The shaded area contains all the stable strain states, and since it is defined by global modes, it coincides with the ellipticity domain.

mode is excluded, the domain for which a bifurcation with mode $\left(\sqrt{s_{1}}, \sqrt{s_{2}}\right)=(0,1)$ is excluded, the domain for which a bifurcation with mode $\left(\sqrt{s_{1}}, \sqrt{s_{2}}\right)=(1,0)$ is excluded, and the domain for which a bifurcation with mode $\left(\sqrt{s_{1}}, \sqrt{s_{2}}\right)=(1,1)$ is excluded. The stability set corresponding to the long wavelength mode (ellipticity domain) is formed by the intersection of the three sectors bounded respectively by the $x_{1}$ axis, the $x_{2}$ axes and the $\Lambda_{1}(\theta)$ curve; the $x_{1}$ axis, the $x_{2}$ axes and the $\Lambda_{2}(\theta)$ curve; and the $x_{1}$ axis, the $x_{2}$ axes and the $\Lambda_{y}(\theta)$ curve (see for example Fig. $3 \mathrm{~b}$ ). In addition to the aforementioned ellipticity domain, one has to consider the three additional sectors bounded respectively by the $x_{1}$ axis, the $x_{2}$ axes and the $\hat{i}(0,1 ; \theta)$ curve; the $x_{1}$ axis, the $x_{2}$ axes and the $\hat{\lambda}(1,0 ; \theta)$ curve; and the $x_{1}$ axis, the $x_{2}$ axes and the $\hat{\lambda}(1,1 ; \theta)$ curve. Results for these calculations in the case of the grillage considered in Fig. 3a $\left(\chi_{a}=\pi / 4, c=\sqrt{2}, \chi_{c}=\pi / 4, r=0.2, \chi_{r}=\pi / 4\right)$ for $0 \leqslant \theta \leqslant \pi / 2$, but with two different nonzero values of $g$, are depicted in Fig. 9a $\left(g=1.0 \times 10^{5}\right)$ and Fig. $9 \mathrm{~b}\left(g=3.0 \times 10^{-5}\right)$. Notice that the $\hat{\lambda}(0,1 ; \theta), \hat{\lambda}(1,0 ; \theta)$ and $\hat{\lambda}(1,1 ; \theta)$ curves lie outside of the $\Lambda_{1}(\theta)$ and $\Lambda_{2}(\theta)$ curves for $g=1.0 \times 10^{5}$ and this means that the critical modes for this model are always long wavelength modes. Hence, the 


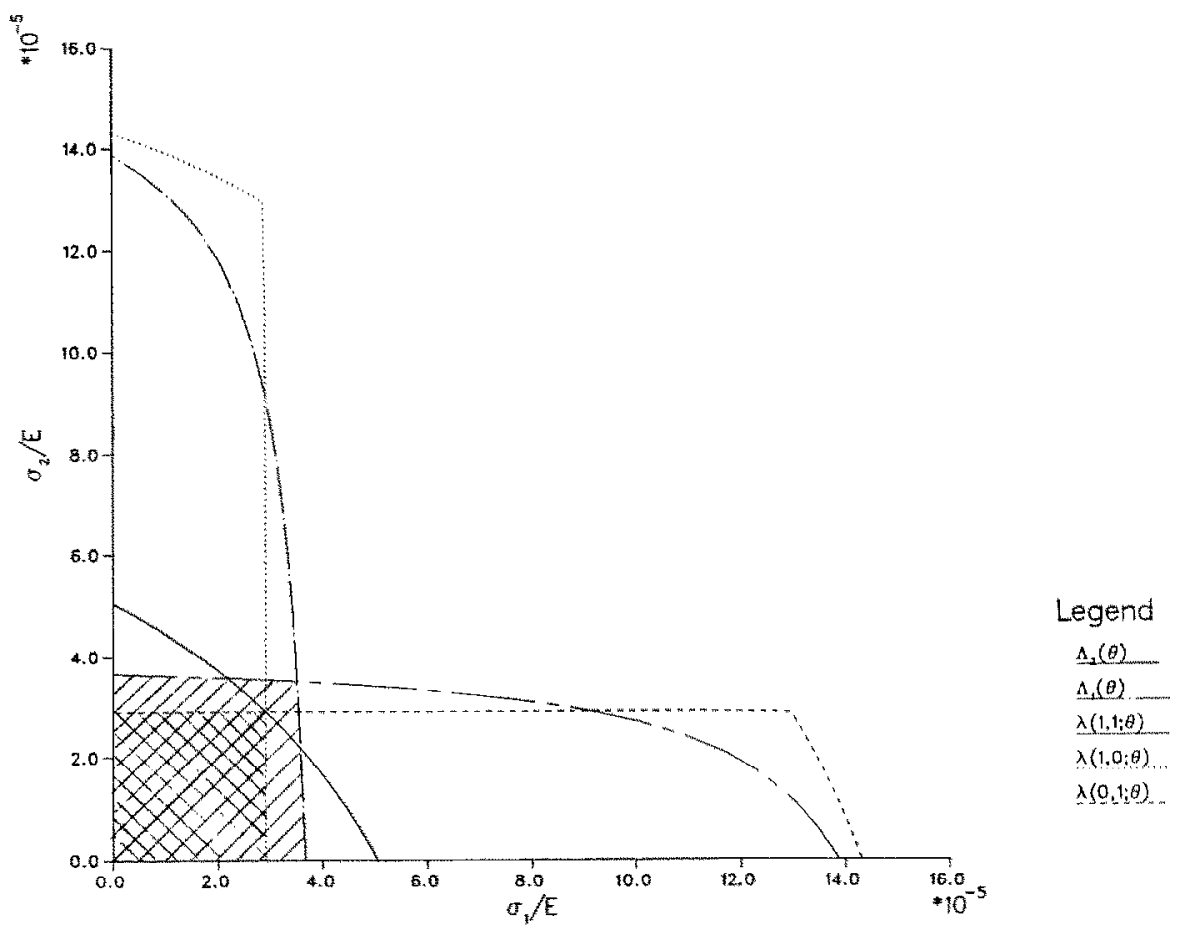

Fro. 9 b. Typical critical dimensionless stress dagram for slender beam grillages with high interstitial simness $q=3.0 \times 10^{-}$. The $(-$,$) and (--\infty)$ hes correspond to the long wavelength mode curves $A_{1}(\theta)$ and $\Lambda_{2}(0)$, while the $(\cdots, \cdots),(\cdots)$ and $(-)$ lines correspond to the finite mode curves $\lambda_{1}(0,1 ; 0), \tilde{A}(1,0 ; 0)$ and $\lambda(1,1 ; 0)$, respectively. The singly shaded area is the ellipticity domain bounded by the $\Lambda_{1}$ and $\Lambda_{2}$ curves, while the doubly shaded area is the stability domain inside of which bifurcation of any wavelength is excluded. Notice that in this case the stability domain is entirely contained inside the ellipticity domain.

ellipticity domain coincides with the stability domain in the $\sigma_{1} / E-\sigma_{2} / E$ space for $g=1.0 \times 10^{-5}$. For the higher interstitial stiffness parameter $g=3.0 \times 10^{-5}$ the situm ation is reversed, as seen in Fig. $9 \mathrm{~b}$, and the stability region is entirely contained inside of the ellipticity domain. For this value of $g$, a local buckling mode will always precede a global one. It is of interest to note that all three curves $\hat{\lambda}(0,1 ; \theta), \hat{\lambda}(1,0 ; \theta)$ and $\hat{\lambda}(1,1 ; \theta)$ intersect at $\sigma_{1} / E=\sigma_{2} / E$ due to the geometry and loading symmetry of this model with respect to the diagonal $x_{1}=x_{2}$.

The dependence of the critical mode on the interstitial stiffness parameter $g$ is depicted in Figs 10a and 10b. Most models, i.e. the ones for which the critical mode occurs at one of the four corners of the $[0,1] \times[0,1]$ domain, generate results very similar to those shown in Fig. $10 \mathrm{a}$, which was calculated for $\chi_{a}=\pi / 4, c=\sqrt{2}$, $\chi_{c}=\pi / 4, r=0.2$ and $\chi_{r}=\pi / 4$, for $\theta=40^{\circ}$. The load ratio parameter was intentionally chosen as $\theta=40^{\circ}$ (as opposed to $\theta=\pi / 4$ ) in order to avoid the situation mentioned above where three modes are critical for $\sigma_{1} / E=\sigma_{2} / E$. As expected, as soon as the interstitial stiffness crosses a critical value, $\sqrt{s_{1}}$ corresponding to the solid line $(-)$ jumps to 1 , while $\sqrt{s_{2}}$ remains at 0 , since the critical mode becomes $\left(\sqrt{s_{1}}, \sqrt{s_{2}}\right)=$ 


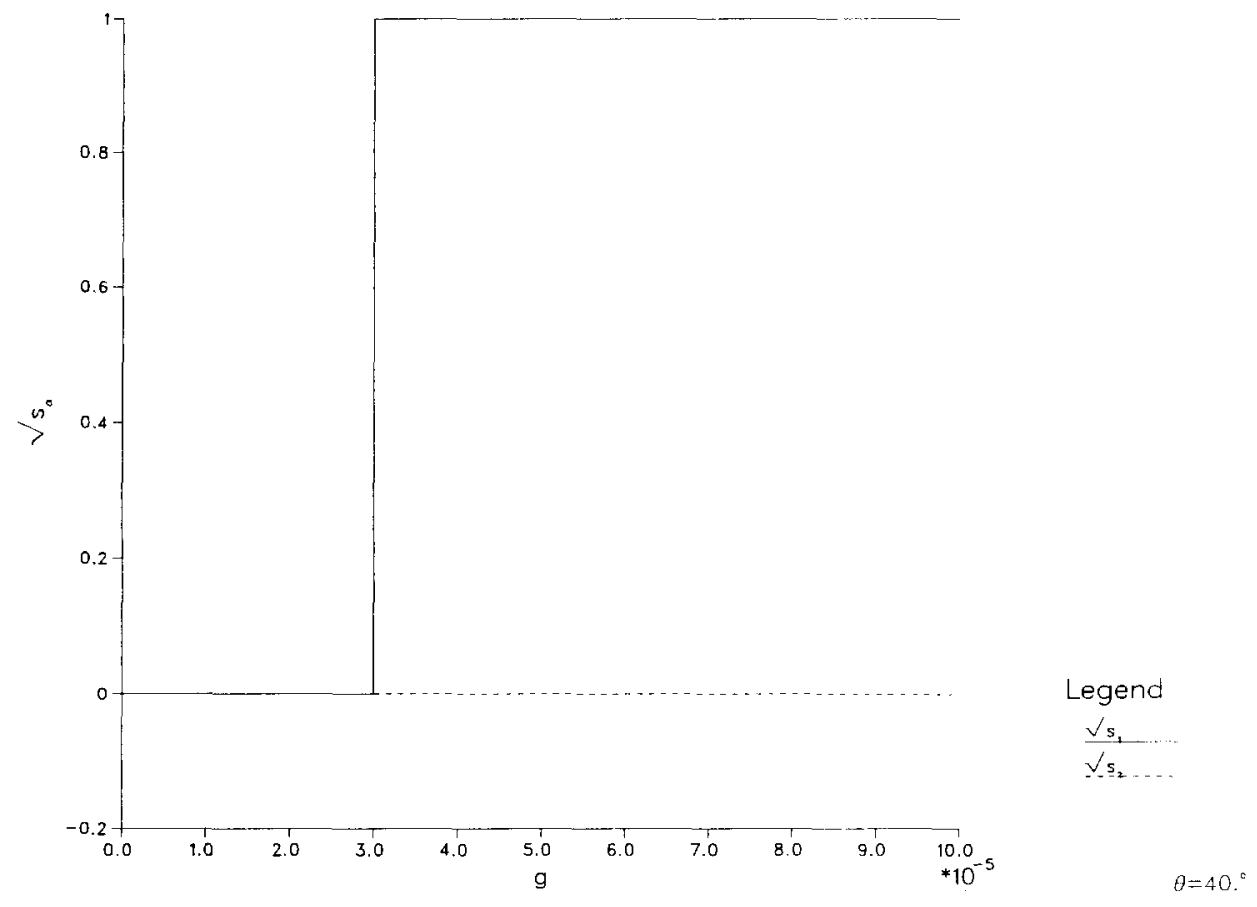

FIG. 10a. Dependence of the eritical wavenumber parameters $\sqrt{s_{1}},(--)$ line, and $\sqrt{s_{2}},(-\ldots)$ line, on the interstitial stiffness $g$ for a slender beam symmetric grillage loaded at $\theta=40$.

$(1,0)$. The more unusual grillages, which are so much stronger in one direction than the other that they can exhibit critical modes in the interior of the $[0,1] \times[0,1]$ domain, all generate results similar to those shown in Fig. 10b. The model used to generate Fig. $10 \mathrm{~b}$ is the same as that used in Fig. $8 \mathrm{~b}\left(\chi_{u}=\pi / 4, c=1.35, \chi_{c}=\pi / 4\right.$, $r=0.1225, \chi_{r}=11.5$ ) for $\theta=0$. In contrast to Fig. 10a, both $\sqrt{ } s_{1}$ and $\sqrt{ } s_{2}$ jump to nontrivial values $(\neq 1, \neq 0)$ simultaneously when $g$ reaches a critical value.

The determining factor for whether or not the critical mode is global or local for a given microstructure is the critical value of the interstitial stiffness parameter, $g_{\mathrm{c}}$. For $0 \leqslant g \leqslant g_{c}$, the critical mode is a global one (determined by the macroscopic homogenized moduli), while for $g>g_{c}$, the critical mode is a local one which is not obtainable from macroscopic information. Consequently, the dependence of $g_{c}$ on the load ratio parameter $\theta$ for a particular grillage is of fundamental importance in characterizing the domains of stable stress states for that grillage. Results of $g_{c}$ as a function of $\theta$ for a number of different slender beam grillages all with $c=\sqrt{ } 2$ and $r=0.20$ are given in Fig. 11. The $y_{r}$ curves were generated using the following four models: one for which $\chi_{a}=42.5, \chi_{c}=\pi / 4$ and $\chi_{r}=\pi / 4,(\cdots)$ line; one for which $\chi_{a}=\pi / 4, \chi_{c}=42.5$ and $\chi_{r}=\pi / 4,(--)$ line; one for which $\chi_{a}=\pi / 4, \chi_{c}=\pi / 4$ and $\chi_{r}=42.5^{\circ},(-\cdot-)$ line; and finally one for which $\chi_{a}=\chi_{c}=\chi_{r}=\pi / 4,(-\longrightarrow)$ line. Note the rather significant variation of $g$, near $\theta=\pi / 4$ in contrast to its relative insensitivity to variation of $\chi_{a}, \chi_{r}$ and $\chi_{r}$ over the rest of the $\theta$ domain. 

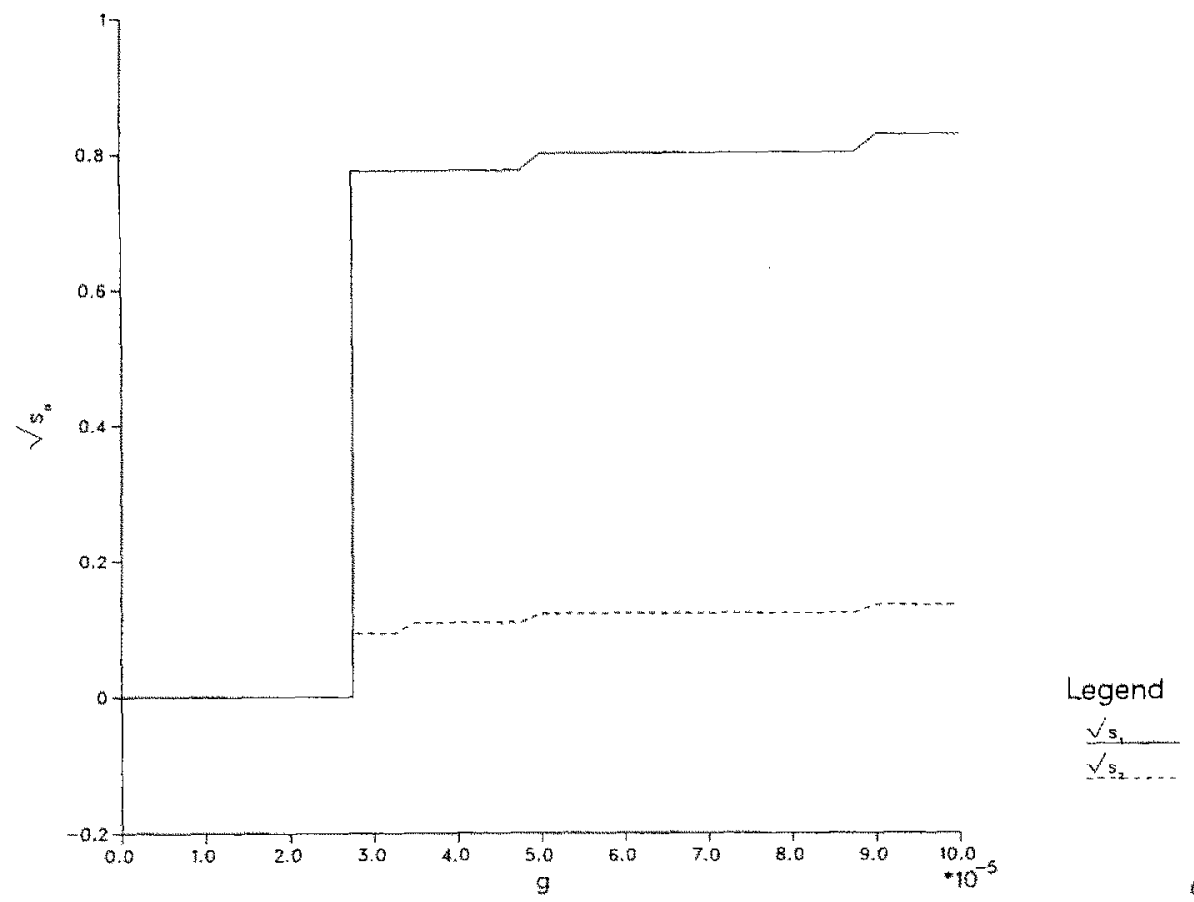

Fic. 10b. Dependence of the critical wavenumber parameters $\sqrt{s_{1}},(-)$ line, and $\sqrt{s_{2}},(--)$ line, on the interstitial stifiness $g$ for the highly nonsymmetrical grillage of Fig, $8 b$ loaded along the stiffer direction $\theta=0$. Notice that in contrast to Fig. $10 \mathrm{a}$, both $\sqrt{s_{1}}$ and $\sqrt{s_{2}}$ are nontrivial functions of $g$.

\section{Conclusions}

Of interest in the present work is the quantitative comparison between the onset of the first instability in a medium with known periodic microstructure and the onset of the corresponding instability predicted by the macroscopic equations for the medium. This comparison establishes the theoretical limits of validity for the averaged (homogenized) response of microstructured elastic media. In the interest of simplicity, attention is focussed on an approximation of a fiber reinforced composite modeled as an infinite periodic grillage of axially compressed beams with an average shear stiffness to approximate the shear resistance effect of the composite's matrix material. The particular beam model was chosen so that the problem would be analytically tractable, yet of sufficient complexity to exhibit nontrivial microscopic and macroscopic failure modes.

A comparison of the stresses at the onset of the microscopic failure (taken to be the first bifurcation away from the principal periodic solution) to the stresses at the onset of the macroscopic failure (which corresponds to the loss of ellipticity for the incremental response of the homogenized model) allows one to identify whether the first failure mode, also termed the critical mode, is local (microscopic critical mode) or global (macroscopic critical mode) in nature. An extensive investigation of the 


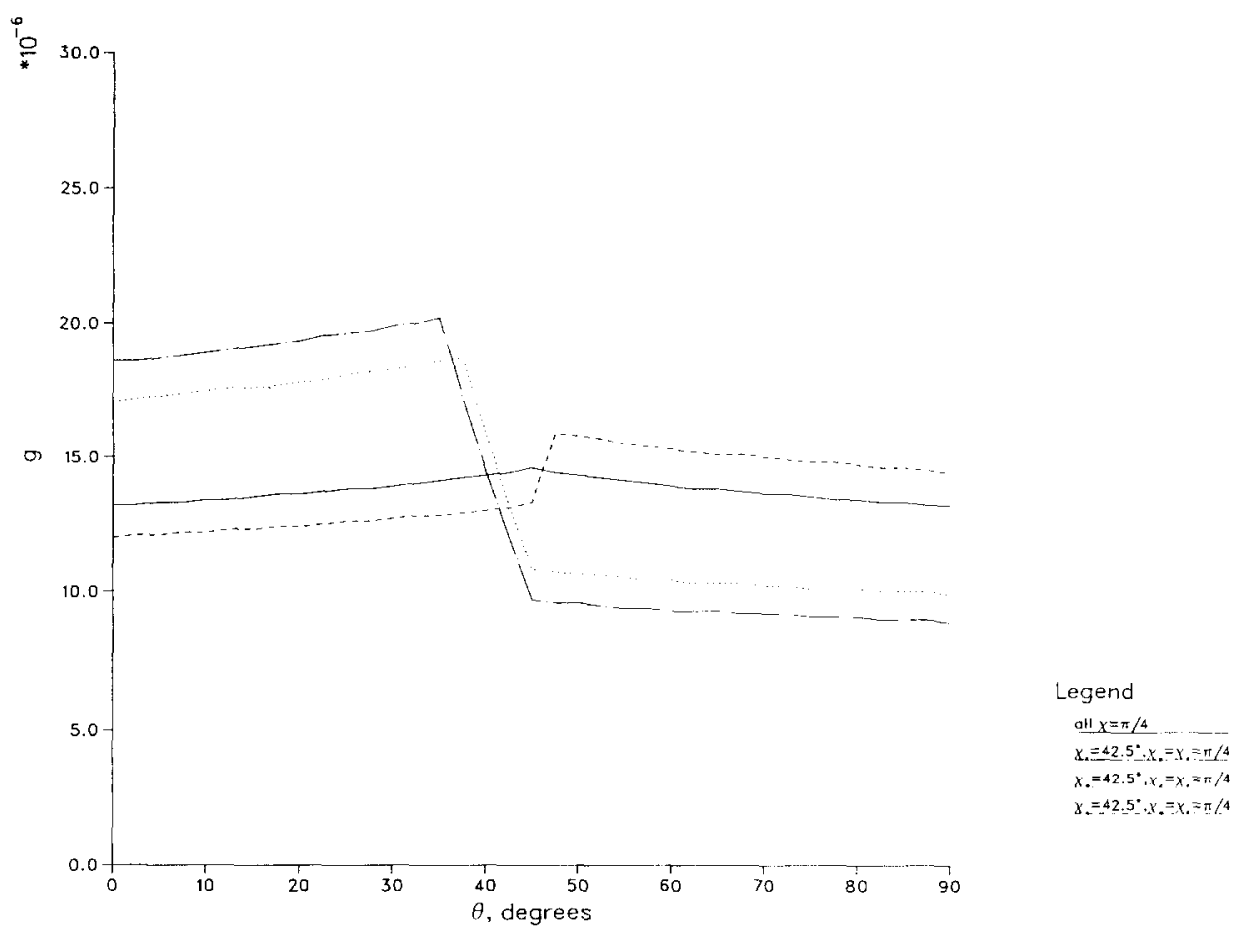

FIG. 11. Dependence of the critical interstitial stiffness parameter $g_{0}$ (below which the long wavelength mode is the critical one and hence the domain of stability is characterized by the ellipticity of the homogenized model) on the loading angle 0 for four different slender beam grillages: $\chi_{a}=\chi_{t}=\chi_{r}=\pi / 4$. $(\cdots)$ line: $\chi_{r}=42.5$ and $\chi_{a}=\chi_{r}=\pi / 4,(\cdots)$ line; $\chi_{a}=42.5$ and $\chi_{c}=\chi_{r}=\pi / 4,(\cdots)$ line: and $\chi_{c}=42.5$ and $\chi_{i i}=\chi_{r}=\pi / 4,(-)$ line.

influence of various model parameters on the failure modes of the composite has been undertaken. The results obtained show the importance of the interstitial stiffness in deciding the nature of the critical mode. For values of the interstitial stiffness below a certain threshold (which depends on the geometry and material properties of the model) the global mode is the critical one which can thus be detected from the homogenized equations of the model. For interstitial stiffnesses above that threshold, a local failure mode precedes the global one, thus rendering the failure predictions of the homogenized model inaccurate. For the case of low interstitial stiffness where the critical mode is global in nature, the stubbiness of the beams plays an important role in the determination of the characteristic direction associated with the loss of ellipticity of the homogenized model. An additional interesting feature of the results is that the critical mode's wavelengths in the $x$-and $y$-directions have no particular relation to the corresponding cell dimensions. Thus, depending on the loading geometry and microstructure, one can have critical modes with wavelengths ranging from any finite number (real, not necessarily integer) of cell lengths (for local critical modes) all the way to modes with wavelengths much larger than the unit cell size (for global critical modes). 
It should be emphasized here that while the above analysis was performed on a fairly specialized periodic medium, it is clear that it can be generalized without difficulty for arbitrary, periodic, three-dimensional, rate independent solids, if one is willing to relax the analytical tractability requirement and work with numerical (f.e.m. usually) techniques. The advantage obviously is that a much richer class of microstructures can be investigated, but the cost is a considerable increase in computer time. A parameter study to determine conditions under which the critical modes are local or global in nature becomes prohibitively time consuming for realistic two- or threedimensional non-degenerate unit cells, as found by TRIANTAFYLLIDIS and MAKER (1987). The present work is thus also useful as an efficient parametric study to determine which loadings and microstructures result in a global critical mode and which result in a local one.

One should keep in mind that in this work failure is defined as the onset of a bifurcation instability in the infinite periodic structure. It is important to determine whether this failure is eatastrophic or not, a question that can be answered only by a postbifurcation analysis of the problem. For the catastrophic postbifurcation solution, the issue of imperfection sensitivity becomes important, for it will provide the load levels permissible in a realistic structure prior to failure. These problems, the solutions of which are the logical continuation of this work, are complicated by the infinite cxtent of the structure and its resulting continuum spectrum for the eigenvalue problem.

The effect of finite boundaries (which have been systematically ignored in the present work) on the response of the composite is another direction for further study. The size of the structure will inevitably enter in the stability analysis for the case where the critical mode is global in nature, i.e. when the critical mode wavelength is much larger than the unit cell size. Boundary effects are also expected even when the critical mode is local in nature, and the interaction between the critical local mode and a global mode which can be present at about the same load level (due to the finite size of the structure) is yet another interesting possibility.

\section{REFERENCES}

Abeyaratne, R. and Triantafyllidis, N. (1984) $J$. Appl. Mech. 51, 481.

ANDERSON, M. S. (1981) AIAA J. 19,782.

ANderson, M. S. and Williams, F. W. (1986) ALAA J. 24, 163.

BALL, J. M. (1977) In Nonlinear Analysis and Mechanics: Heriot-Watt Symposium Proceedings, Vol. I, p. 187. Pitman, London.

BAZ̆ant, Z. P. and Christensen, M. (1972) J. Struct. Engng ASCE 98, 2269.

Bažant, Z. P. and Christensen, M. (1973) Int. J. Engng Sci. 11, 1255.

Bendsoe, M. P. and Triantafyllidis, N. (1990) Int. J. Solids Struct. 26, 725.

Bensoussan, A., Lions, J. L. and Papanicolaou, G. (1978) Asymptotic Analysis for Periodic Siructures. North-Holland, Amsterdam.

Forman, S. and Hutchinson, J. W. (1970) Int. J. Solids Struct. 6, 909.

Geymonat, G., Müller, S. and Triantafyllidis, N. (1990) C.R. Acad. Sci. Paris 311, S I, 911.

Geymonat, G., Müller, S. and Triantafyllidis, N. (1992) Arch. Rat. Mech. Anal., to appear.

LiveSLeY, R. K. (1968) Matrix Methods in Structural Analysis. Pergamon Press, Oxford. 
Rice, J. R. (1976) In Theoretical and Applied Mechanics (ed. W. T. KoITER), p. 207. NorthHolland, Amsterdam.

Triantafyllidis, N. and MAKER, B. N. (1985) J. Appl. Mech. 52, 794.

Triantafyllidis, N. and Maker, B. N. (1987) unpublished work.

TVERGAARD, V. (1981) Int. J. Fracture 17, 389.

WAH, T. (1965) J. Mech. Phys. Solids 13, 1.

\section{APPENDIX}

The calculation of $\mathbf{K}_{x \beta}^{J}$ for beams under axial loading follows a procedure exposed in Livesi.r. (1968). The expression for the total potential energy for a beam $J \mathscr{E}_{\text {neam, }}$, is given in (2). By solving the equilibrium equations $\delta \mathscr{E}_{\mathrm{heam}}=0$, one can express $\mathscr{E}_{\mathrm{beam}}$ in terms of prescribed end (elemental) displacements and rotations $\hat{\mathbf{d}}_{x}$. (Note that the hat notation is used here to differentiate an end nodal displacement and rotation vector $\hat{\mathbf{d}}_{x}$ in local coordinates from its counterpart, $\mathbf{d}_{i}$, expressed in global coordinates, referred to earlier.) The elemental incremental stiffness matrices $\hat{\mathbf{K}}_{z \beta}^{s}$ for the beam in local coordinates are then given by

$$
\hat{\mathbf{K}}_{x / \beta}^{\prime}=\frac{\hat{\partial}^{2} \mathscr{E}_{\mathrm{heam},}}{\partial \hat{\mathbf{d}}_{z} \hat{\partial \mathbf{d}_{\beta}}}
$$

For the case of the beam loaded by a compressive axial force $N_{J}$, this procedure yields the following elemental incremental stiffness matrices $\hat{\mathbf{K}}_{\not \not \beta}^{J}$ along the principal equilibrium path:

where*

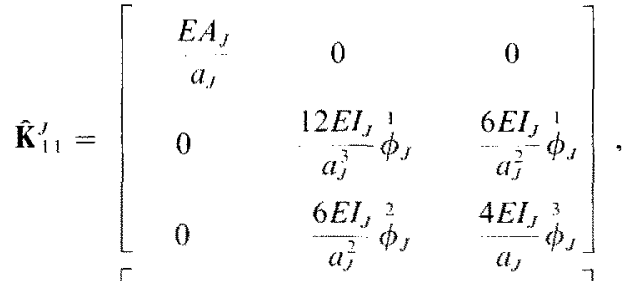

$$
\begin{aligned}
& \hat{\mathbf{K}}_{12}^{J}=\left[\begin{array}{ccc}
E A_{J} & 0 & 0 \\
-a_{J} & 12 E I_{J} & 6 E I_{J}^{2} \\
0 & -\frac{a_{J}^{3}}{a_{J}^{3}} & \frac{a_{J}^{2}}{\phi_{J}} \\
0 & -\frac{6 E I_{J}^{2}}{a_{J}^{2}} \phi_{J} & 2 E I_{J}^{4} \\
a_{J}
\end{array}\right] \text {, } \\
& \hat{\mathbf{K}}_{21}^{3}=\left[\begin{array}{ccc}
-\frac{E A_{J}}{a_{l}} & 0 & 0 \\
0 & -\frac{12 E I_{J}}{a_{J}^{3}} \phi_{j} & -6 E I_{J}^{2} \\
& 6 E I_{J}^{2} \phi_{j}^{2} & 2 E I_{J}{ }^{4} \\
0 & a_{j}^{2} \phi_{J} & a_{J}
\end{array}\right], \\
& \hat{\mathbf{K}}_{22}^{J}=\left[\begin{array}{ccc}
E A_{J} & 0 & 0 \\
a_{J} & \frac{12 E I_{J}}{a_{J}^{3}} \phi_{J} & -\frac{6 E I_{J}}{a_{J}^{2}} \phi_{J} \\
0 & -\frac{6 E I_{J}}{a_{J}^{2}} \phi_{J} & -\frac{4 E I_{J}}{a_{J}} \dot{\phi}_{J}
\end{array}\right],
\end{aligned}
$$

* Note that the subscript $\alpha$ is used in (A.3) because the quantities described there are the same for beams 1 and 3 and are the same for beams 2 and 4 . This is in contrast to (A.2) where the subscript $J$ is retained. $J$ is used there because the four beams that make up the unit cell have four different gemetric orientations. 


$$
\begin{aligned}
& \stackrel{\mathrm{l}}{\phi_{\alpha}} \equiv\left(\sigma_{\alpha}+\tau_{\alpha}\right) / 6-\beta_{\alpha}^{2} / 12, \quad \stackrel{2}{\phi}_{\alpha} \equiv\left(\sigma_{\alpha}+\tau_{\alpha}\right) / 6, \quad \stackrel{3}{\phi_{\alpha}} \equiv \sigma_{\alpha} / 4, \quad \stackrel{4}{\phi}_{\alpha} \equiv \tau_{\alpha} / 2, \\
& \sigma_{\alpha} \equiv \beta_{x}\left(\sin \left[\beta_{\alpha}\right]-\beta_{\alpha} \cos \left[\beta_{\alpha}\right]\right) /\left\{2\left(1-\cos \left[\beta_{\alpha}\right]\right)-\beta_{\alpha} \sin \left[\beta_{\alpha}\right]\right\} \text {, } \\
& \tau_{\alpha} \equiv \beta_{x}\left(\beta_{\alpha}-\sin \left[\beta_{\alpha}\right]\right) /\left\{2\left(1-\cos \left[\beta_{\alpha}\right]\right)-\beta_{x} \sin \left[\beta_{\alpha}\right]\right\} \text {, } \\
& \beta_{\alpha}^{2} \equiv N_{x} a_{x}^{2} /\left(E_{\alpha} I_{\alpha}\right) \text {. }
\end{aligned}
$$

The above results hold for the case of the beam axis aligned with the $x_{1}$ direction, since they provide elemental incremental stiffness matrices. In order to obtain the global incremental stiffness matrices $\mathbf{K}_{z \beta}^{J}$ for a beam of given $\hat{\mathbf{K}}_{z \beta}^{J}$ matrices and orientation angle $\gamma$ with respect to the $x_{1}$ axis, the following transformation is used.

$$
\mathbf{K}_{\alpha \beta \beta}^{J}=\mathbf{T} \hat{\mathbf{K}}_{\alpha \beta}^{J} \mathbf{T}^{\mathrm{T}} .
$$

The orientation matrix $\mathbf{T}$ is

$$
\mathbf{T}=\left[\begin{array}{ccc}
\cos \gamma & -\sin \gamma & 0 \\
\sin \gamma & \cos \gamma & 0 \\
0 & 0 & 1
\end{array}\right]
$$

The calculation of the $\mathbf{L}_{l}$ is similar to but more straightforward than the above procedure for obtaining the $\mathbf{K}_{\alpha \beta}^{J}$, because the internal strain energy $\mathscr{E}_{\text {interstital }}$ for the matrix material approximation is already in terms of global nodal displacement quantities (and is quadratic in those quantities)

$$
\mathbf{L}_{l J}=\frac{\partial^{2} \mathscr{E}_{\text {interstitial }}}{\partial \mathbf{d}_{I} \partial \mathbf{d}_{J}}
$$

The $\mathbf{L}_{l j}$ are

$$
\begin{aligned}
& \mathbf{L}_{11}=-\mathbf{L}_{13}=-\mathbf{L}_{31}=\mathbf{L}_{33}=\left[\begin{array}{ccc}
0 & 0 & 0 \\
0 & G a_{2} / a_{1} & 0 \\
0 & 0 & 0
\end{array}\right], \\
& \mathbf{L}_{12}=-\mathbf{L}_{14}=-\mathbf{L}_{32}=\mathbf{L}_{34}=\left[\begin{array}{lll}
0 & 0 & 0 \\
G & 0 & 0 \\
0 & 0 & 0
\end{array}\right], \\
& \mathbf{L}_{21}=-\mathbf{L}_{23}=-\mathbf{L}_{41}=\mathbf{L}_{43}=\left[\begin{array}{lll}
0 & G & 0 \\
0 & 0 & 0 \\
0 & 0 & 0
\end{array}\right], \\
& \mathbf{L}_{22}=-\mathbf{L}_{24}=-\mathbf{L}_{42}=\mathbf{L}_{44}=\left[\begin{array}{ccc}
G a_{1} / a_{2} & 0 & 0 \\
0 & 0 & 0 \\
0 & 0 & 0
\end{array}\right] .
\end{aligned}
$$

